\title{
HYBRID FINITE ELEMENT MODELS FOR PIEZOELECTRIC MATERIALS*
}

\author{
K.Y.Sze \\ Department of Mechanical Engineering, The University of Hong Kong \\ Pokfulam Road, Hong Kong SAR, P.R.CHINA
}

Y.S.Pan

Institute of Computational Engineering Sciences

Southwest Jiaotong University, Chengdu 610031, P.R.CHINA

\begin{abstract}
In this paper, hybrid variational principles are employed for piezoelectric finite element formulation. Starting from eight-node hexahedral elements with displacement and electric potential as the nodal d.of.s, hybrid models with assumed stress and electric displacement are devised. The assumed stress and electric displacement are chosen to be contravariant with the minimal eighteen and seven modes, respectively. The pertinent coefficients can be condensed in the element level and do not enter the system equation. A number of benchmark tests are exercised. The predicted results indicate that the assumed stress and electric displacements are effectively in improving the element accuracy.
\end{abstract}

* this work was conducted when the second author was visiting the University of Hong Kong as a research associate

Published in Journal of Sound and Vibration (1999) 226(3), 519-547 


\section{INTRODUCTION}

Piezoelectric materials have been indispensable for electromechanical resonators, transducers, sensors, actuators and adaptive structures. Owing to the complexity of the governing equations in piezoelectricity, only a few simple problems such as simply supported beams and plates can be solved analytically [1-4]. Since Allik \& Hughes [5] presented their work on finite element (f.e.) method for piezoelectric vibration analysis, the method has been the dominating practical tool for design and analysis of piezoelectric devices and adaptive structures. Inheriting Allik \& Hughes' work, all of the f.e. models presented in references [6-22] include displacement and electric potential as the only assumed field variables. Other fields such as stress, electric displacement etc. are derived from displacement and electric potential. These models and the associated formulation can be classified as irreducible in the sense that the number of field variables cannot be further reduced [23]. Same as the irreducible or displacement elements in structural mechanics, irreducible piezoelectric elements are often too stiff, susceptible to mesh distortion and aspect ratio. To overcome these drawbacks, Tzou \& Tseng [14], Ha, Keilers \& Chang [16] and Tzou [17] made use of bubble/incompatible displacement modes [24,25] to improve the eight-node hexahedral element.

In addition to bubble/incompatible displacement method, hybrid (or reducible) variational principles in structural mechanics have been successfully employed for enhancing the element accuracy and circumventing various locking phenomena [26-41]. In this light, Ghandi \& Hagood [42] have proposed a piezoelectric hybrid tetrahedral finite element model in which electric displacement, electric potential and displacement are assumed. Their model is markedly superior to the irreducible model. Besides reference [42], hybrid variational principles have rarely been used in formulating piezoelectric finite element models.

In this paper, we shall start with a general hybrid variational principle that contains stress, strain, displacement, electric displacement, electric field and electric potential as the independently assumed field variables. It will be seen that the stationary conditions of the functional are the nine governing equations in linear piezoelectricity. For domain decomposition methods such as f.e. method, the prerequisites and the continuity requirements on the field variables assumed in the principle are addressed. Four degenerated versions of the general principle are adopted for f.e. formulation. Judging from the results obtained for a number of benchmark problems, the proposed hybrid models are more accurate than the irreducible ones. 
For a solid piezoelectric body occupying domain $\Omega$, the governing equations are summarized below $[43,44]$ :

(i) strain-displacement relation: $\boldsymbol{\gamma}=\mathbf{D}_{m} \mathbf{u}$ in $\Omega$

(ii) electric field-electric potential relation : $\mathbf{E}=-\mathbf{D}_{e} \phi$ in

(iii) constitutive relations : $\left\{\begin{array}{l}\boldsymbol{\tau} \\ \mathbf{D}\end{array}\right\}=\left[\begin{array}{cc}\mathbf{c}_{E} & -\mathbf{e}^{T} \\ \mathbf{e} & \boldsymbol{\epsilon}_{\gamma}\end{array}\right]\left\{\begin{array}{l}\boldsymbol{\gamma} \\ \mathbf{E}\end{array}\right\}$ in $\Omega$

(iv) stress equilibrium condition : $\mathbf{D}_{m}^{T} \boldsymbol{\tau}+\overline{\mathbf{b}}=\mathbf{0} \quad$ in $\Omega$

(v) charge conservation condition : $\mathbf{D}_{e}^{T} \mathbf{D}=\mathbf{0}$ in $\Omega$,

(vi) mechanical natural boundary condition : $\mathbf{n}_{m} \boldsymbol{\tau}=\overline{\mathbf{t}}$ on $\mathrm{S}_{t}$

(vii) electric natural boundary condition : $\mathbf{n}_{e} \mathbf{D}=\bar{\omega}$ on $\mathrm{S}_{\omega}$,

(viii) mechanical essential boundary condition : $\mathbf{u}=\overline{\mathbf{u}}$ on $\mathrm{S}_{u}$,

(ix) electric essential boundary condition : $\phi=\bar{\phi} \quad$ on $\mathrm{S}_{\phi}$

where

$\boldsymbol{\gamma}=\left\{\gamma_{x x}, \gamma_{y y}, \gamma_{z z}, 2 \gamma_{x y}, 2 \gamma_{y z}, 2 \gamma_{z x}\right\}^{T}$ is the vector of strain components

$\mathbf{u}=\left\{u_{x}, u_{y}, u_{z}\right\}^{T}$ is the displacement, $\mathbf{E}=\left\{E_{x}, E_{y}, E_{z}\right\}^{T}$ is the electric field,

$\boldsymbol{\tau}=\left\{\tau_{x x}, \tau_{y y}, \tau_{z z}, \tau_{x y}, \tau_{y z}, \tau_{z x}\right\}^{T}$ is the vector of stress components,

$\mathbf{D}=\left\{D_{x}, D_{y}, D_{z}\right\}^{T}$ is the electric displacement, $\overline{\mathbf{b}}=\left\{\bar{b}_{x}, \bar{b}_{y}, \bar{b}_{z}\right\}^{T}$ is the body force

$\overline{\mathbf{t}}=\left\{\bar{t}_{x}, \bar{t}_{y}, \bar{t}_{z}\right\}^{T}$ is the prescribed traction, $\overline{\mathbf{u}}=\left\{\bar{u}_{x}, \bar{u}_{y}, \bar{u}_{z}\right\}^{T}$ is the prescribed displacement

$\mathbf{c}_{E}=\mathbf{c}_{E}^{T}$ is the elasticity matrix measured at constant electric field

$\mathbf{e}$ is the piezoelectric matrix measured at measured at constant strain

$\boldsymbol{\epsilon}_{\gamma}=\boldsymbol{\epsilon}_{\gamma}^{T}$ is the dielectric matrix measured at constant strain

$\mathbf{D}_{e}=\left[\begin{array}{l}\partial / \partial x \\ \partial / \partial y \\ \partial / \partial z\end{array}\right], \mathbf{D}_{m}=\left[\begin{array}{cccccc}\partial / \partial x & 0 & 0 & \partial / \partial y & 0 & \partial / \partial z \\ 0 & \partial / \partial y & 0 & \partial / \partial x & \partial / \partial z & 0 \\ 0 & 0 & \partial / \partial z & 0 & \partial / \partial y & \partial / \partial x\end{array}\right]^{T}$

$\mathbf{n}_{m}=\left[\begin{array}{cccccc}n_{x} & 0 & 0 & n_{y} & 0 & n_{z} \\ 0 & n_{y} & 0 & n_{x} & n_{z} & 0 \\ 0 & 0 & n_{z} & 0 & n_{y} & n_{x}\end{array}\right], \mathbf{n}_{e}=\left[\begin{array}{ccc}n_{x} & 0 & 0 \\ 0 & n_{y} & 0 \\ 0 & 0 & n_{z}\end{array}\right]$

$\left\{n_{x}, n_{y}, n_{z}\right\}^{T}$ is the unit outward normal vector to the boundary $\partial \Omega$ of domain $\Omega$ 
It will be assumed as usual that the boundary $\partial \Omega$ of the domain can be partitioned according to the boundary conditions into $\mathrm{S}_{t}, \mathrm{~S}_{u}, \mathrm{~S}_{\omega}$ and $\mathrm{S}_{\phi}$ such that

$$
\mathrm{S}_{t} \cap \mathrm{S}_{u}=\mathrm{S}_{\omega} \cap \mathrm{S}_{\phi}=\text { null }, \mathrm{S}_{t} \cup \mathrm{S}_{u}=\mathrm{S}_{\omega} \cup \mathrm{S}_{\phi}=\partial \Omega
$$

It is noteworthy that $\boldsymbol{\gamma}$ and $-\mathbf{E}$ are the energy conjugates of $\boldsymbol{\tau}$ and $\mathbf{D}$, respectively. By changing the objects in the constitutive relations, the following alternate forms can be obtained :

$$
\begin{aligned}
& \left\{\begin{array}{l}
\boldsymbol{\gamma} \\
\mathbf{E}
\end{array}\right\}=\left[\begin{array}{cc}
\mathbf{c}_{E} & -\mathbf{e}^{T} \\
\mathbf{e} & \boldsymbol{\epsilon}_{\varepsilon}
\end{array}\right]^{-1}\left\{\begin{array}{l}
\boldsymbol{\tau} \\
\mathbf{D}
\end{array}\right\}=\left[\begin{array}{ll}
\mathbf{s}_{D} & \mathbf{g}^{T} \\
-\mathbf{g} & \mathbf{f}_{\sigma}
\end{array}\right]\left\{\begin{array}{l}
\boldsymbol{\tau} \\
\mathbf{D}
\end{array}\right\} \\
& \left\{\begin{array}{l}
\boldsymbol{\tau} \\
\mathbf{E}
\end{array}\right\}=\left[\begin{array}{cc}
\mathbf{c}_{E}+\mathbf{e}^{T} \mathbf{\epsilon}_{\gamma}^{-1} \mathbf{e} & -\left(\boldsymbol{\epsilon}_{\gamma}^{-T} \mathbf{e}\right)^{T} \\
-\boldsymbol{\epsilon}_{\gamma}^{-T} \mathbf{e} & \mathbf{f}_{\gamma}
\end{array}\right]\left\{\begin{array}{l}
\boldsymbol{\gamma} \\
\mathbf{D}
\end{array}\right\}=\left[\begin{array}{cc}
\mathbf{c}_{D} & -\mathbf{h}^{T} \\
-\mathbf{h} & \mathbf{f}_{\gamma}
\end{array}\right]\left\{\begin{array}{l}
\boldsymbol{\gamma} \\
\mathbf{D}
\end{array}\right\}
\end{aligned}
$$

\section{A GENERAL VARIATIONAL PRINCIPLE FOR PIEZOELECTRICITY}

A few researchers have investigated the variational principles for piezoelectric bodies $[43,44]$. The most general variational principle that includes all the six assumed field variables is :

$$
\begin{aligned}
\Pi_{G}= & \int_{\Omega}\left[\frac{1}{2}\left\{\begin{array}{c}
\boldsymbol{\gamma} \\
-\mathbf{E}
\end{array}\right\}^{T}\left[\begin{array}{cc}
\mathbf{c}_{E} & \mathbf{e}^{T} \\
\mathbf{e} & -\boldsymbol{\epsilon}_{\gamma}
\end{array}\right]\left\{\begin{array}{c}
\boldsymbol{\gamma} \\
-\mathbf{E}
\end{array}\right\}-\left\{\begin{array}{c}
\boldsymbol{\tau} \\
\mathbf{D}
\end{array}\right\}^{T}\left(\left\{\begin{array}{c}
\boldsymbol{\gamma} \\
-\mathbf{E}
\end{array}\right\}-\left\{\begin{array}{c}
\mathbf{D}_{m} \mathbf{u} \\
\mathbf{D}_{e} \phi
\end{array}\right\}\right)-\overline{\mathbf{b}}^{T} \mathbf{u}\right] d v-\int_{S_{t}} \overline{\mathbf{t}}^{T} \mathbf{u} d s-\int_{S_{\omega}} \phi \bar{\omega} d s \\
& -\int_{S_{u}}\left(\mathbf{n}_{m} \boldsymbol{\tau}\right)^{T}(\mathbf{u}-\overline{\mathbf{u}}) d s-\int_{S_{\phi}}\left(\mathbf{n}_{e} \mathbf{D}\right)^{T}(\phi-\bar{\phi}) d s
\end{aligned}
$$

where

$$
\frac{1}{2}\left\{\begin{array}{c}
\boldsymbol{\gamma} \\
-\mathbf{E}
\end{array}\right\}^{T}\left[\begin{array}{cc}
\mathbf{c}_{E} & \mathbf{e}^{T} \\
\mathbf{e} & -\boldsymbol{\epsilon}_{\gamma}
\end{array}\right]\left\{\begin{array}{c}
\boldsymbol{\gamma} \\
-\mathbf{E}
\end{array}\right\}=H(\boldsymbol{\gamma},-\mathbf{E})
$$

is known as the electric enthalpy. By recalling the divergence theorems, we have

$$
\begin{aligned}
& \int_{\Omega}\left(\delta \mathbf{u} \mathbf{D}{ }_{m}^{T} \boldsymbol{\tau}+\boldsymbol{\tau}^{T} \mathbf{D}_{m} \delta \mathbf{u}\right) d v=\int_{\partial \Omega}\left(\mathbf{n}_{m} \boldsymbol{\tau}\right)^{T} \delta \mathbf{u} d s \\
& \int_{\Omega}\left(\delta \phi \mathbf{D}{ }_{e}^{T} \mathbf{D}+\mathbf{D}^{T} \mathbf{D}{ }_{e} \delta \phi\right) d v=\int_{\partial \Omega}\left(\mathbf{n}_{e} \mathbf{D}\right)^{T} \delta \phi d s
\end{aligned}
$$

in which $\delta$ is the variational symbol. Variation of $\Pi_{\mathrm{G}}$ can then be worked out :

$$
\left.\left.\delta \Pi_{G}=\int_{\Omega}\left[\begin{array}{c}
\delta \boldsymbol{\gamma} \\
-\delta \mathbf{E}
\end{array}\right\}^{T}\left(\begin{array}{cc}
\mathbf{c}_{E} & \mathbf{e}^{T} \\
\mathbf{e} & -\boldsymbol{\epsilon}_{\gamma}
\end{array}\right]\left\{\begin{array}{c}
\boldsymbol{\gamma} \\
-\mathbf{E}
\end{array}\right\}-\left\{\begin{array}{c}
\boldsymbol{\tau} \\
\mathbf{D}
\end{array}\right\}\right)-\left\{\begin{array}{c}
\delta \boldsymbol{\tau} \\
\delta \mathbf{D}
\end{array}\right\}^{T}\left(\left\{\begin{array}{c}
\boldsymbol{\gamma} \\
-\mathbf{E}
\end{array}\right\}-\left\{\begin{array}{c}
\mathbf{D}_{m} \mathbf{u} \\
\mathbf{D}_{e} \phi
\end{array}\right\}\right)-\left\{\begin{array}{c}
\delta \mathbf{u} \\
\delta \phi
\end{array}\right\}^{T}\left\{\begin{array}{c}
\mathbf{D}{ }_{m}^{T} \boldsymbol{\tau}+\overline{\mathbf{b}} \\
\mathbf{D}_{e}^{T} \mathbf{D}
\end{array}\right\}\right] d v
$$




$$
+\int_{S_{t}}\left(\mathbf{n}_{e} \boldsymbol{\tau}-\overline{\mathbf{t}}\right)^{T} \delta \mathbf{u} d s+\int_{S_{\omega}}\left(\mathbf{n}_{e} \mathbf{D}-\bar{\omega}\right) \delta \phi d s-\int_{S_{u}}\left(\mathbf{n}_{m} \delta \boldsymbol{\tau}\right)^{T}(\mathbf{u}-\overline{\mathbf{u}}) d s-\int_{S_{\phi}}\left(\mathbf{n}_{e} \delta \mathbf{D}\right)^{T}(\phi-\bar{\phi}) d s
$$

The generalization of the functional can be seen as its Euler's equations includes all the nine governing equations in Eqn.(1).

\section{DOMAIN DECOMPOSITION}

We now consider the piezoelectric domain $\Omega$ be decomposed into two subdomains $\Omega^{1}$ and $\Omega^{2}$ as shown in Fig.1. Let superscripts be used for subdomain designation and $S_{12}$ be the subdomain interfacial for $\Omega^{1}$ and $\Omega^{2}$, it will be assumed that

$$
S_{t}^{1} \cup S_{t}^{2}=S_{t}, S_{u}^{1} \cup S_{u}^{2}=S_{u}, S_{\omega}^{1} \cup S_{\omega}^{2}=S_{\omega}, S_{\phi}^{1} \cup S_{\phi}^{2}=S_{\phi}
$$

Moreover,

$$
\Omega^{1} \cup \Omega^{2}=\Omega, S_{t}^{i} \cup S_{u}^{i} \cup S_{12}=S_{\omega}^{i} \cup S_{\phi}^{i} \cup S_{12}=\partial \Omega^{i} \quad \text { for } i=1,2
$$

The governing equations after decomposing the domain are :
(i) $\boldsymbol{\gamma}^{i}=\mathbf{D}_{m} \mathbf{u}^{i} \quad$ in $\Omega^{i}$,
(ii) $\mathbf{E}^{i}=-\mathbf{D} \phi_{e}^{i}$ in $\Omega^{i}$,
(iii) $\left\{\begin{array}{c}\boldsymbol{\tau}^{i} \\ \mathbf{D}^{i}\end{array}\right\}=\left[\begin{array}{c}\mathbf{c}_{E}^{i} \\ \mathbf{e}^{i}\end{array}\right.$
$\left.\begin{array}{c}-\left(\mathbf{e}^{i}\right)^{T} \\ \boldsymbol{\epsilon}_{\gamma}\end{array}\right]\left\{\begin{array}{c}\boldsymbol{\gamma}^{i} \\ \mathbf{E}^{i}\end{array}\right\}$ in $\Omega^{i}$
(iv) $\mathbf{D}_{m}^{T} \boldsymbol{\tau}^{i}+\overline{\mathbf{b}}=\mathbf{0}$ in $\Omega^{i},(\mathrm{v})$
$\mathbf{D}{ }_{e}^{T} \mathbf{D}^{i}=\mathbf{0}$ in $\Omega^{i}$
(vi) $\mathbf{n}_{m}^{i} \boldsymbol{\tau}^{i}=\overline{\mathbf{t}} \quad$ on $S_{t}^{i}$
(vii) $\mathbf{n}_{e}^{i} \mathbf{D}^{i}=\bar{\omega} \quad$ on $S_{\omega}^{i}$
(viii) $\mathbf{u}^{i}=\overline{\mathbf{u}} \quad$ on $S_{u}^{i}$,
(ix) $\phi^{i}=\bar{\phi}$
on $S_{\phi}^{i}$

(x) mechanical reciprocity condition : $\mathbf{n}_{m}^{1} \boldsymbol{\tau}^{1}+\mathbf{n}_{m}^{2} \boldsymbol{\tau}^{2}=\mathbf{0}$

on $S_{12}$

(xi) electric reciprocity condition :

$$
\mathbf{n}_{e}^{1} \mathbf{D}^{1}+\mathbf{n}_{e}^{2} \mathbf{D}^{2}=0
$$

on $S_{12}$

(xii) mechanical compatibility condition : $\quad \mathbf{u}^{1}=\mathbf{u}^{2}$

on $S_{12}$

(xiii) electric compatibility condition : $\quad \phi^{1}=\phi^{2}$

on $S_{12}$

for $i=1$ and 2. Compared to Eqn.(1), there are four extra conditions to be satisfied on the subdomain interface $S_{12}$. With $\Omega=\Omega^{1} \cup \Omega^{2}, \Pi_{G}$ in Eqn.(4) can be expressed as :

$$
\Pi_{G}=\Pi_{G}^{1}+\Pi_{G}^{2}
$$

where

$$
\begin{aligned}
\Pi_{G}^{i}= & \left.\int_{\Omega^{i}}\left[\frac{1}{2}\left\{\begin{array}{c}
\boldsymbol{\gamma}^{i} \\
-\mathbf{E}^{i}
\end{array}\right\}^{T}\left[\begin{array}{cc}
\mathbf{c}_{E}^{i} & \left(\mathbf{e}^{i}\right)^{T} \\
\mathbf{e}^{i} & -\boldsymbol{\epsilon}_{\gamma}^{i}
\end{array}\right]\left\{\begin{array}{c}
\boldsymbol{\gamma}^{i} \\
-\mathbf{E}^{i}
\end{array}\right\}-\left\{\begin{array}{c}
\boldsymbol{\tau}^{i} \\
\mathbf{D}^{i}
\end{array}\right\}^{T}\left\{\begin{array}{c}
\boldsymbol{\gamma}^{i} \\
-\mathbf{E}^{i}
\end{array}\right\}-\left\{\begin{array}{l}
\mathbf{D}_{m} \mathbf{u}^{i} \\
\mathbf{D}_{e} \phi^{i}
\end{array}\right\}\right)-(\overline{\mathbf{b}})^{T} \mathbf{u}^{i}\right] d v \\
& -\int_{S_{t}^{i}} \overline{\mathbf{t}}^{T} \mathbf{u}^{i} d s-\int_{S_{\omega}^{i}} \phi^{i} \bar{\omega} d s-\int_{S_{u}^{i}}\left(\mathbf{n}_{m}^{i} \boldsymbol{\tau}^{i}\right)^{T}\left(\mathbf{u}^{i}-\overline{\mathbf{u}}\right) d s-\int_{S_{\phi}^{i}}\left(\mathbf{n}_{e}^{i} \mathbf{D}^{i}\right)^{T}\left(\phi^{i}-\bar{\phi}\right) d s
\end{aligned}
$$


By invoking Eqn.(5) and Eqn.(7),

$$
\begin{gathered}
\left.\left.\delta \Pi_{G}=\sum_{i=1}^{2}\left(\int_{\Omega^{i}}\left[\begin{array}{c}
\delta \boldsymbol{\gamma}^{i} \\
-\delta \mathbf{E}^{i}
\end{array}\right\}^{T}\left[\begin{array}{cc}
\mathbf{c}_{E}^{i} & \left(\mathbf{e}^{i}\right)^{T} \\
\mathbf{e}^{i} & -\boldsymbol{\epsilon}_{\gamma}^{i}
\end{array}\right]\left\{\begin{array}{c}
\boldsymbol{\gamma}^{i} \\
-\mathbf{E}^{i}
\end{array}\right\}-\left\{\begin{array}{l}
\boldsymbol{\tau}^{i} \\
\mathbf{D}^{i}
\end{array}\right\}\right)-\left\{\begin{array}{c}
\delta \boldsymbol{\tau}^{i} \\
\delta \mathbf{D}^{i}
\end{array}\right\}^{T}\left(\begin{array}{c}
\boldsymbol{\gamma}^{i} \\
-\mathbf{E}^{i}
\end{array}\right\}-\left\{\begin{array}{l}
\mathbf{D}_{m} \mathbf{u}^{i} \\
\mathbf{D}_{e} \phi^{i}
\end{array}\right\}\right)\right] d v \\
-\int_{\Omega^{i}}\left\{\begin{array}{c}
\delta \mathbf{u}^{i} \\
\delta \phi^{i}
\end{array}\right\}^{T}\left\{\begin{array}{c}
\mathbf{D}{ }_{m}^{T} \boldsymbol{\tau}^{i}-\overline{\mathbf{b}} \\
\mathbf{D}_{e}^{T} \mathbf{D}^{i}
\end{array}\right\} d v+\int_{S_{t}^{i}}\left(\mathbf{n}_{m}^{i} \boldsymbol{\tau}^{i}-\overline{\mathbf{t}}\right)^{T} \delta \mathbf{u}^{i} d s+\int_{S_{\omega}^{i}}\left(\mathbf{n}_{e}^{i} \mathbf{D}^{i}-\bar{\omega}\right) \delta \phi^{i} d s \\
\left.-\int_{S_{u}^{i}}\left(\mathbf{n}_{m}^{i} \delta \boldsymbol{\tau}^{i}\right)^{T}\left(\mathbf{u}^{i}-\overline{\mathbf{u}}\right) d s-\int_{S_{\phi}^{i}}\left(\mathbf{n}_{e}^{i} \delta \mathbf{D}^{i}\right)^{T}\left(\phi^{i}-\bar{\phi}\right) d s+\int_{S_{12}}\left[\left(\mathbf{n}_{m}^{i} \boldsymbol{\tau}^{i}\right)^{T} \delta \mathbf{u}^{i}+\left(\mathbf{n}_{e}^{i} \mathbf{D}^{i}\right) \delta \phi^{i}\right] d s\right)
\end{gathered}
$$

By constraining the two compatibility conditions, we have

$$
\mathbf{u}^{1}=\mathbf{u}^{2}, \delta \mathbf{u}^{1}=\delta \mathbf{u}^{2}, \phi^{1}=\phi^{2} \text { and } \delta \phi^{1}=\delta \phi^{2} \quad \text { on } S_{12}
$$

with which the last term in $\delta \Pi_{G}$ can be expressed as :

$$
\sum_{i=1}^{2} \int_{S_{12}}\left[\left(\mathbf{n}_{m}^{i} \boldsymbol{\tau}^{i}\right)^{T} \delta \mathbf{u}^{i}+\left(\mathbf{n}_{e}^{i} \mathbf{D}^{i}\right) \delta \phi^{i}\right] d s=\int_{S_{12}}\left[\left(\mathbf{n}_{m}^{1} \boldsymbol{\tau}^{1}+\mathbf{n}_{m}^{2} \boldsymbol{\tau}^{2}\right)^{T} \delta \mathbf{u}^{1}+\left(\mathbf{n}_{e}^{1} \mathbf{D}^{1}+\mathbf{n}_{e}^{2} \mathbf{D}^{2}\right) \delta \phi^{1}\right] d s
$$

Hence, with the two compatibility conditions satisfied as a priori, Euler's equations of $\Pi_{G}$ include the first eleven conditions in Eqn.(8). In other words, zeroth order continuity of the displacement and electric potential at the subdomain interface must be ensured when $\Pi_{G}$ is employed. There is no continuity requirement on the other field variables at the subdomain interface, i.e. the two sets of the field variables in the two subdomains can be totally independent of each other. The arguments presented here can readily be generalized to multiply subdomains such as in f.e. meshes.

\section{DEGENERATED VARIATIONAL PRINCIPLES}

In f.e. method, the two essential boundary conditions can be satisfied by having displacement and electric potential as the nodal variables. With the two conditions constrained, $\Pi_{\mathrm{G}}$ is simplified to :

$$
\left.\Pi_{m G}=\int_{\Omega}\left[\frac{1}{2}\left\{\begin{array}{c}
\boldsymbol{\gamma} \\
-\mathbf{E}
\end{array}\right\}^{T}\left[\begin{array}{cc}
\mathbf{c}_{E} & \mathbf{e}^{T} \\
\mathbf{e} & -\boldsymbol{\epsilon}_{\gamma}
\end{array}\right]\left\{\begin{array}{c}
\boldsymbol{\gamma} \\
-\mathbf{E}
\end{array}\right\}-\left\{\begin{array}{c}
\boldsymbol{\tau} \\
\mathbf{D}
\end{array}\right\}^{T}\left(\begin{array}{c}
\boldsymbol{\gamma} \\
-\mathbf{E}
\end{array}\right\}-\left\{\begin{array}{c}
\mathbf{D}{ }_{m} \mathbf{u} \\
\mathbf{D}_{e} \phi
\end{array}\right\}\right)-\overline{\mathbf{b}}^{T} \mathbf{u}\right] d v-\int_{S_{t}}^{\mathbf{t}^{T}} \mathbf{u} d s-\int_{S_{\omega}} \phi \bar{\omega} d s
$$

In this paper, four variation functionals degenerated from $\Pi_{m G}$ will be employed for finite element formulation. 
I. Functional with only $\mathbf{u}$ and $\phi$ Assumed - With the electric field-potential relation $\mathbf{E}=-\mathbf{D} \phi_{e}$ and the strain-displacement relation $\boldsymbol{\gamma}=\mathbf{D}_{m} \mathbf{u}$ constrained, the assumed stress, strain, electric field and electric displacement can be eliminated from $\Pi_{m G}$. The resulting functional is :

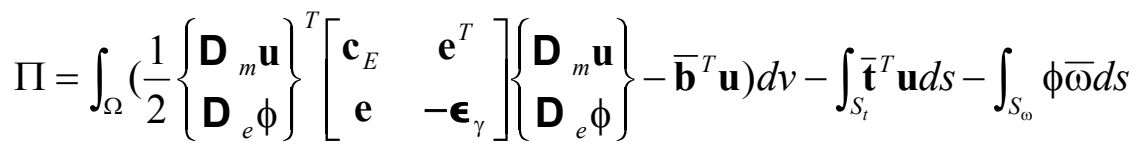

This gives rise to the irreducible formulation in piezoelectricity [5,23].

II. Functional with $\mathbf{D}$, u and $\phi$ Assumed - With the strain-displacement relation $\boldsymbol{\gamma}=\mathbf{D}_{m} \mathbf{u}$ and the constitutive relation $\mathbf{D}=\mathbf{e} \boldsymbol{\gamma}+\boldsymbol{\epsilon}_{\gamma} \mathbf{E}$ constrained, the assumed stress, strain and electric field can be eliminated from $\Pi_{m G}$. The resulting functional is :

$$
\Pi_{D}=\int_{\Omega}\left[\frac{1}{2}\left\{\begin{array}{c}
\mathbf{D}{ }_{m} \mathbf{u} \\
\mathbf{D}
\end{array}\right\}^{T}\left[\begin{array}{cc}
\mathbf{c}_{D} & -\mathbf{h}^{T} \\
-\mathbf{h} & \mathbf{f}_{\gamma}
\end{array}\right]\left\{\begin{array}{c}
\mathbf{D}_{m} \mathbf{u} \\
\mathbf{D}
\end{array}\right\}+\mathbf{D}^{T} \mathbf{D}_{e} \phi-\overline{\mathbf{b}}^{T} \mathbf{u}\right] d v-\int_{S_{t}} \overline{\mathbf{t}}^{T} \mathbf{u} d s-\int_{S_{\omega}} \phi \bar{\omega} d s
$$

III. Functional with $\boldsymbol{\tau}$, $\mathbf{u}$ and $\phi$ Assumed - With the electric field-electric potential relation $\mathbf{E}=-\mathbf{D}_{e} \phi$ and the constitutive relation $\boldsymbol{\tau}=\mathbf{c}_{E} \boldsymbol{\gamma}-\mathbf{e}^{T} \mathbf{E}$ constrained, strain, electric field and electric displacement can be eliminated from $\Pi_{m G}$. The resulting functional is :

$$
\Pi_{\tau}=\int_{\Omega}\left(\frac{-1}{2}\left\{\begin{array}{c}
\boldsymbol{\tau} \\
\mathbf{D} \\
e
\end{array}\right\}^{T}\left[\begin{array}{cc}
\mathbf{s}_{E} & -\mathbf{d}^{T} \\
-\mathbf{d} & \boldsymbol{\epsilon}_{\tau}
\end{array}\right]\left\{\begin{array}{c}
\boldsymbol{\tau} \\
\mathbf{D} \\
e
\end{array}\right\}+\boldsymbol{\tau}^{T} \mathbf{D}_{m} \mathbf{u}-\overline{\mathbf{b}}^{T} \mathbf{u}\right) d v-\int_{S_{t}} \overline{\mathbf{t}}^{T} \mathbf{u} d s-\int_{S_{\omega}} \phi \bar{\omega} d s
$$

$I V$. Functional with $\boldsymbol{\tau}, \mathbf{D}$, u and $\phi$ Assumed - With the constitutive relations $\boldsymbol{\tau}=\mathbf{c}_{E} \boldsymbol{\gamma}-\mathbf{e}^{T} \mathbf{E}$ and $\mathbf{D}=\mathbf{e} \boldsymbol{\gamma}+\boldsymbol{\epsilon}_{\gamma} \mathbf{E}$ constrained, the assumed strain and electric field can be eliminated from $\Pi_{m G}$. The resulting functional is :

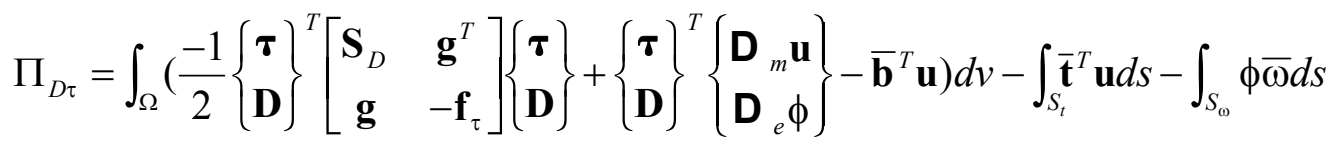

where

$$
\frac{1}{2}\left\{\begin{array}{c}
\boldsymbol{\tau} \\
\mathbf{D}
\end{array}\right\}^{T}\left[\begin{array}{cc}
\mathbf{S}_{D} & \mathbf{g}^{T} \\
\mathbf{g} & -\mathbf{f}_{\tau}
\end{array}\right]\left\{\begin{array}{l}
\boldsymbol{\tau} \\
\mathbf{D}
\end{array}\right\}=M(\boldsymbol{\tau}, \mathbf{D}) \quad \text { is known as the mechanical enthalpy. }
$$

Only assumed stress and/or electric displacement are considered in Eqn.(15) to Eqn.(17) in addition to assumed displacement and electric potential because the homogenous equilibrium and charge 
conservation conditions can readily be satisfied by manipulating the stress and electric displacement shape functions.

\section{FINITE ELEMENT FORMULATION}

Being degenerated version of $\Pi_{G}$, the prerequisites and continuity requirements on the field variables of $\Pi, \Pi_{D}, \Pi_{\tau}$ and $\Pi_{D \tau}$ are identical to that discussed in Section 4 . In other words, the two compatibility conditions must be satisfied as priori whereas the assumed stress and electric displacement in each element can be independent to the ones in other elements. Zeroth order continuity of the displacement and electric potential can be met by having displacement and electric potential as the nodal d.o.f.s.

In the Section 4, superscripts are employed for subdomain designation. Superscripts of the field variables will here be dropped for simplicity. Within a generic element, the assumed field variables are discretized as :

$$
\mathbf{u}=\mathbf{N}_{m} \mathbf{q}_{m}, \phi=\mathbf{N}_{e} \mathbf{q}_{e}, \boldsymbol{\tau}=\mathbf{P}_{m} \boldsymbol{\beta}_{m}, \mathbf{D}=\mathbf{P}_{e} \boldsymbol{\beta}_{e}
$$

in which $\mathbf{N}_{m}$ is the displacement interpolation matrix, $\mathbf{q}_{m}$ is the vector of element nodal displacement d.o.f.s, $\mathbf{N}_{e}$ is the electric potential interpolation matrix, $\mathbf{q}_{e}$ is the vector of element nodal electric potential d.o.f.s., $\mathbf{P}_{m}$ is the stress shape function matrix, $\boldsymbol{\beta}_{m}$ is the vector of stress coefficients, $\mathbf{P}_{e}$ is the electric displacement shape function matrix and $\boldsymbol{\beta}_{e}$ is the vector of electric displacement coefficients. Moreover, we define

$$
\mathbf{B}_{m}=\mathbf{D}_{m} \mathbf{N}_{m}, \mathbf{B}_{e}=\mathbf{D} \mathbf{N}_{e}
$$

It has been shown that $\boldsymbol{\tau}$ and $\mathbf{D}$ need not be continuous across the element interface. Thus, every element has its own coefficient vectors $\boldsymbol{\beta}_{e}$ and $\boldsymbol{\beta}_{m}$ which can be condensed in the element level.

Finite Element Formulation using $\Pi=\sum_{e} \Pi^{e}-$ The elementwise version of $\Pi$ is :

$$
\Pi^{e}=\int_{\Omega^{e}}\left(\frac{1}{2}\left\{\begin{array}{l}
\mathbf{D}_{m} \mathbf{u} \\
\mathbf{D}_{e} \phi
\end{array}\right\}^{T}\left[\begin{array}{cc}
\mathbf{c}_{E} & \mathbf{e}^{T} \\
\mathbf{e} & -\boldsymbol{\epsilon}_{\gamma}
\end{array}\right]\left\{\begin{array}{l}
\mathbf{D}_{m} \mathbf{u} \\
\mathbf{D}_{e} \phi
\end{array}\right\}-\overline{\mathbf{b}}^{T} \mathbf{u}\right) d v-P_{S}^{e}
$$

where $\Omega^{e}$ denotes the element domain and

$$
P_{S}^{e}=\int_{S_{t}^{e}} \overline{\mathbf{t}}^{T} \mathbf{u} d s+\int_{S_{\omega}^{e}} \phi \bar{\omega} d s
$$


denotes the surface load acting on the element. With Eqn.(18) and Eqn.(19) invoked :

$$
\begin{aligned}
& \Pi^{e}=\frac{1}{2}\left\{\begin{array}{l}
\mathbf{q}_{m} \\
\mathbf{q}_{e}
\end{array}\right\}^{T} \mathbf{k}^{e}\left\{\begin{array}{l}
\mathbf{q}_{m} \\
\mathbf{q}_{e}
\end{array}\right\}-\left\langle\overline{\mathbf{b}}^{T} \mathbf{N}_{m}\right\rangle \mathbf{q}_{m}-P_{S}^{e} \\
& \mathbf{k}^{e}=\left[\begin{array}{cc}
\left\langle\mathbf{B}_{m}^{T} \mathbf{c}_{E} \mathbf{B}_{m}\right\rangle & \left\langle\mathbf{B}_{m}^{T} \mathbf{e}^{T} \mathbf{B}_{e}\right\rangle \\
\left\langle\mathbf{B}_{e}^{T} \mathbf{e B}_{m}\right\rangle & -\left\langle\mathbf{B}_{e}^{T} \mathbf{\epsilon}_{\gamma} \mathbf{B}_{e}\right\rangle
\end{array}\right] \text { is the element matrix }
\end{aligned}
$$

Finite Element Formulation using $\Pi_{D}=\sum_{e} \Pi_{D}^{e}$ - The elementwise version of $\Pi_{\mathrm{D}}$ is :

$$
\Pi_{D}^{e}=\int_{\Omega^{e}}\left[\frac{1}{2}\left\{\begin{array}{c}
\mathbf{D} \\
\mathbf{D}^{\mathbf{u}}
\end{array}\right\}^{T}\left[\begin{array}{cc}
\mathbf{c}_{D} & -\mathbf{h}^{T} \\
-\mathbf{h} & \mathbf{f}_{\gamma}
\end{array}\right]\left\{\begin{array}{c}
\mathbf{D}{ }_{m} \mathbf{u} \\
\mathbf{D}
\end{array}\right\}+\mathbf{D}^{T} \mathbf{D}_{e} \phi-\overline{\mathbf{b}}^{T} \mathbf{u}\right] d v-P_{S}^{e}
$$

With Eqn.(18) and Eqn.(19) invoked :

$$
\Pi_{D}^{e}=\frac{1}{2}\left\{\begin{array}{l}
\mathbf{q}_{m} \\
\boldsymbol{\beta}_{e}
\end{array}\right\}^{T}\left[\begin{array}{cc}
\left\langle\mathbf{B}_{m}^{T} \mathbf{c}_{D} \mathbf{B}_{m}\right\rangle & -\left\langle\mathbf{B}_{m}^{T} \mathbf{h}^{T} \mathbf{P}_{e}\right\rangle \\
-\left\langle\mathbf{P}_{e}^{T} \mathbf{h} \mathbf{B}_{m}\right\rangle & \left\langle\mathbf{P}_{e}^{T} \mathbf{f}_{\gamma} \mathbf{P}_{e}\right\rangle
\end{array}\right]\left\{\begin{array}{l}
\mathbf{q}_{m} \\
\boldsymbol{\beta}_{e}
\end{array}\right\}+\boldsymbol{\beta}_{e}^{T} \mathbf{B}_{e} \mathbf{q}_{e}-\left\langle\overline{\mathbf{b}}^{T} \mathbf{N}_{m}\right\rangle \mathbf{q}_{m}-P_{S}^{e}
$$

Variation of $\boldsymbol{\beta}_{e}$ results in :

$$
\boldsymbol{\beta}_{e}=\left[\begin{array}{ll}
\left\langle\mathbf{P}_{e}^{T} \mathbf{h} \mathbf{B}_{m}\right\rangle & -\left\langle\mathbf{P}_{e}^{T} \mathbf{B}_{e}\right\rangle
\end{array}\right]\left\{\begin{array}{l}
\mathbf{q}_{m} \\
\mathbf{q}_{e}
\end{array}\right\}
$$

with which

$$
\begin{aligned}
& \Pi_{D}^{e}=\frac{1}{2}\left\{\begin{array}{l}
\mathbf{q}_{m} \\
\mathbf{q}_{e}
\end{array}\right\}^{T} \mathbf{k}_{D}^{e}\left\{\begin{array}{l}
\mathbf{q}_{m} \\
\mathbf{q}_{e}
\end{array}\right\}-\left\langle\overline{\mathbf{b}}^{T} \mathbf{N}_{m}\right\rangle \mathbf{q}_{m}-P_{S}^{e} \\
& \mathbf{k}_{D}^{e}=\left[\begin{array}{cc}
\left\langle\mathbf{B}_{m}^{T} \mathbf{c}_{D} \mathbf{B}_{m}\right\rangle & \mathbf{0} \\
\mathbf{0} & \mathbf{0}
\end{array}\right]-\left[\begin{array}{l}
\left\langle\mathbf{P}_{e}^{T} \mathbf{h} \mathbf{B}_{m}\right\rangle^{T} \\
-\left\langle\mathbf{P}_{e}^{T} \mathbf{B}_{e}\right\rangle^{T}
\end{array}\right]\left\langle\mathbf{P}_{e}^{T} \mathbf{f}_{\gamma} \mathbf{P}_{e}\right\rangle^{-1}\left[\left\langle\mathbf{P}_{e}^{T} \mathbf{h} \mathbf{B}_{m}\right\rangle-\left\langle\mathbf{P}_{e}^{T} \mathbf{B}_{e}\right\rangle\right]
\end{aligned}
$$

Finite Element Formulation using $\Pi_{\tau}=\sum_{e} \Pi_{\tau}^{e}$ - The elementwise version of $\Pi_{\tau}$ is :

$$
\Pi_{\tau}^{e}=\int_{\Omega^{e}}\left(\frac{-1}{2}\left\{\begin{array}{c}
\boldsymbol{\tau} \\
\mathbf{D}_{e} \phi
\end{array}\right\}^{T}\left[\begin{array}{cc}
\mathbf{s}_{E} & -\mathbf{d}^{T} \\
-\mathbf{d} & \boldsymbol{\epsilon}_{\tau}
\end{array}\right]\left\{\begin{array}{c}
\boldsymbol{\tau} \\
\mathbf{D}_{e} \phi
\end{array}\right\}+\boldsymbol{\tau}^{T} \mathbf{D}_{m} \mathbf{u}-\overline{\mathbf{b}}^{T} \mathbf{u}\right) d v-P_{S}^{e}
$$

With Eqn.(18) and Eqn.(19) invoked :

$$
\Pi_{\tau}^{e}=\frac{-1}{2}\left\{\begin{array}{c}
\boldsymbol{\beta}_{m} \\
\mathbf{q}_{e}
\end{array}\right\}^{T}\left[\begin{array}{cc}
\left\langle\mathbf{P}_{m}^{T} \mathbf{s}_{E} \mathbf{P}_{m}\right\rangle & -\left\langle\mathbf{P}_{m}^{T} \mathbf{d}^{T} \mathbf{B}_{e}\right\rangle \\
-\left\langle\mathbf{B}_{e}^{T} \mathbf{d} \mathbf{P}_{m}\right\rangle & \left\langle\mathbf{B}_{e}^{T} \mathbf{\epsilon}_{\tau} \mathbf{B}_{e}\right\rangle
\end{array}\right]\left\{\begin{array}{c}
\boldsymbol{\beta}_{m} \\
\mathbf{q}_{e}
\end{array}\right\}+\boldsymbol{\beta}_{m}^{T}\left\langle\mathbf{P}_{m}^{T} \mathbf{B}_{m}\right\rangle \mathbf{q}_{m}-\left\langle\overline{\mathbf{b}}^{T} \mathbf{N}_{m}\right\rangle \mathbf{q}_{m}-P_{S}^{e}
$$


Variation of $\boldsymbol{\beta}_{m}$ results in :

$$
\left.\boldsymbol{\beta}_{m}=\left\langle\mathbf{P}_{m}^{T} \mathbf{s}_{E} \mathbf{P}_{m}\right\rangle^{-1}\left[\left\langle\mathbf{P}_{m}^{T} \mathbf{B}_{m}\right\rangle\left\langle\mathbf{P}_{m}^{T} \mathbf{d}^{T} \mathbf{B}_{e}\right\rangle\right]\right]\left\{\begin{array}{c}
\mathbf{q}_{m} \\
\mathbf{q}_{e}
\end{array}\right\}
$$

with which

$$
\begin{aligned}
& \Pi_{\tau}^{e}=\frac{1}{2}\left\{\begin{array}{l}
\mathbf{q}_{m} \\
\mathbf{q}_{e}
\end{array}\right\}^{T} \mathbf{k}_{\tau}^{e}\left\{\begin{array}{l}
\mathbf{q}_{m} \\
\mathbf{q}_{e}
\end{array}\right\}-\left\langle\overline{\mathbf{b}}^{T} \mathbf{N}_{m}\right\rangle \mathbf{q}_{m}-P_{S}^{e} \\
& \mathbf{k}_{\tau}^{e}=\left[\begin{array}{c}
\left\langle\mathbf{P}_{m}^{T} \mathbf{B}_{m}\right\rangle^{T} \\
\left\langle\mathbf{P}_{m}^{T} \mathbf{d}^{T} \mathbf{B}_{e}\right\rangle^{T}
\end{array}\right]\left\langle\mathbf{P}_{m}^{T} \mathbf{s}_{E} \mathbf{P}_{m}\right\rangle^{-1}\left[\left\langle\mathbf{P}_{m}^{T} \mathbf{B}_{m}\right\rangle\left\langle\mathbf{P}_{m}^{T} \mathbf{d}^{T} \mathbf{B}_{e}\right\rangle\right]-\left[\begin{array}{cc}
\mathbf{0} & \mathbf{0} \\
\mathbf{0} & \left\langle\mathbf{B}_{e}^{T} \mathbf{\epsilon}_{\tau} \mathbf{B}_{e}\right\rangle
\end{array}\right]
\end{aligned}
$$

Finite Element Formulation using $\Pi_{\tau D}=\sum_{e} \Pi_{\tau D}^{e}-$ The elementwise version of $\Pi_{\tau \mathrm{D}}$ is :

$$
\Pi_{\tau D}^{e}=\int_{\Omega^{e}}\left(\frac{-1}{2}\left\{\begin{array}{l}
\boldsymbol{\tau} \\
\mathbf{D}
\end{array}\right\}^{T}\left[\begin{array}{cc}
\mathbf{S}_{D} & \mathbf{g}^{T} \\
\mathbf{g} & -\mathbf{f}_{\tau}
\end{array}\right]\left\{\begin{array}{l}
\boldsymbol{\tau} \\
\mathbf{D}
\end{array}\right\}+\left\{\begin{array}{l}
\boldsymbol{\tau} \\
\mathbf{D}
\end{array}\right\}^{T}\left\{\begin{array}{l}
\mathbf{D}_{m} \mathbf{u} \\
\mathbf{D}_{e} \phi
\end{array}\right\}-\overline{\mathbf{b}}^{T} \mathbf{u}\right) d v-P_{S}^{e}
$$

With Eqn.(18) and Eqn.(19) invoked :

$$
\begin{aligned}
& \Pi_{\tau D}^{e}=\frac{-1}{2}\left\{\begin{array}{l}
\boldsymbol{\beta}_{m} \\
\boldsymbol{\beta}_{e}
\end{array}\right\}^{T}\left[\begin{array}{cc}
\left\langle\mathbf{P}_{m}^{T} \mathbf{S}_{D} \mathbf{P}_{m}\right\rangle & \left\langle\mathbf{P}_{m}^{T} \mathbf{g}^{T} \mathbf{P}_{e}\right\rangle \\
\left\langle\mathbf{P}_{e}^{T} \mathbf{g P}_{m}\right\rangle & -\left\langle\mathbf{P}_{e}^{T} \mathbf{f}_{\sigma} \mathbf{P}_{e}\right\rangle
\end{array}\right]\left\{\begin{array}{l}
\boldsymbol{\beta}_{m} \\
\boldsymbol{\beta}_{e}
\end{array}\right\}+\left\{\begin{array}{l}
\boldsymbol{\beta}_{m} \\
\boldsymbol{\beta}_{e}
\end{array}\right\}^{T}\left[\begin{array}{cc}
\left\langle\mathbf{P}_{m}^{T} \mathbf{B}_{m}\right\rangle & \mathbf{0} \\
\mathbf{0} & \left\langle\mathbf{P}_{e}^{T} \mathbf{B}_{e}\right\rangle
\end{array}\right]\left\{\begin{array}{l}
\mathbf{q}_{m} \\
\mathbf{q}_{e}
\end{array}\right\}_{m} \\
& -\left\langle\overline{\mathbf{b}}^{T} \mathbf{N}_{m}\right\rangle \mathbf{q}_{m}-P_{S}^{e}
\end{aligned}
$$

Variation of $\boldsymbol{\beta}_{m}$ and $\boldsymbol{\beta}_{e}$ results in :

$$
\left\{\begin{array}{l}
\boldsymbol{\beta}_{m} \\
\boldsymbol{\beta}_{e}
\end{array}\right\}=\left[\begin{array}{cc}
\left\langle\mathbf{P}_{m}^{T} \mathbf{S}_{D} \mathbf{P}_{m}\right\rangle & \left\langle\mathbf{P}_{m}^{T} \mathbf{g}^{T} \mathbf{P}_{e}\right\rangle \\
\left\langle\mathbf{P}_{e}^{T} \mathbf{g} \mathbf{P}_{m}\right\rangle & -\left\langle\mathbf{P}_{e}^{T} \mathbf{f}_{\sigma} \mathbf{P}_{e}\right\rangle
\end{array}\right]^{-1}\left[\begin{array}{cc}
\left\langle\mathbf{P}_{m}^{T} \mathbf{B}_{m}\right\rangle & \mathbf{0} \\
\mathbf{0} & \left\langle\mathbf{P}_{e}^{T} \mathbf{B}_{e}\right\rangle
\end{array}\right]\left\{\begin{array}{l}
\mathbf{q}_{m} \\
\mathbf{q}_{e}
\end{array}\right\}
$$

with which

$$
\begin{aligned}
& \Pi_{\tau D}^{e}=\frac{1}{2}\left\{\begin{array}{l}
\mathbf{q}_{m} \\
\mathbf{q}_{e}
\end{array}\right\}^{T} \mathbf{k}_{\tau D}^{e}\left\{\begin{array}{l}
\mathbf{q}_{m} \\
\mathbf{q}_{e}
\end{array}\right\}-\left\langle\overline{\mathbf{b}}^{T} \mathbf{N}_{m}\right\rangle \mathbf{q}_{m}-P_{S}^{e} \\
& \mathbf{k}_{\tau D}^{e}=\left[\begin{array}{cc}
\left\langle\mathbf{P}_{m}^{T} \mathbf{B}_{m}\right\rangle & \mathbf{0} \\
\mathbf{0} & \left\langle\mathbf{P}_{e}^{T} \mathbf{B}_{e}\right\rangle
\end{array}\right]\left[\begin{array}{cc}
\left\langle\mathbf{P}_{m}^{T} \mathbf{S}_{D} \mathbf{P}_{m}\right\rangle & \left\langle\mathbf{P}_{m}^{T} \mathbf{g}^{T} \mathbf{P}_{e}\right\rangle \\
\left\langle\mathbf{P}_{e}^{T} \mathbf{g} \mathbf{P}_{m}\right\rangle & -\left\langle\mathbf{P}_{e}^{T} \mathbf{f}_{\sigma} \mathbf{P}_{e}\right\rangle
\end{array}\right]^{-1}\left[\begin{array}{cc}
\left\langle\mathbf{P}_{m}^{T} \mathbf{B}_{m}\right\rangle & \mathbf{0} \\
\mathbf{0} & \left\langle\mathbf{P}_{e}^{T} \mathbf{B}_{e}\right\rangle
\end{array}\right]
\end{aligned}
$$




\section{DETERMINATION OF EIGEN FREQUENCIES}

In eigen frequency analysis, the surface loads vanish and the inertial force can be incorporated as the body force, i.e. $\overline{\mathbf{b}}=-\rho \ddot{\mathbf{u}}$. Similar to the conventional eigen frequency analysis, we assume

$$
\mathbf{u}=\widetilde{\mathbf{u}} e^{i \theta t}, \phi=\widetilde{\phi} e^{i \theta t}, \boldsymbol{\tau}=\widetilde{\boldsymbol{\tau}} e^{i \theta t}, \mathbf{D}=\widetilde{\mathbf{D}} e^{i \theta t} \text { and thus } \ddot{\mathbf{u}}=-\theta^{2} \mathbf{u}
$$

where quantities with over-tiles denote their amplitudes, $t$ is time and $\theta$ is the eigen frequency. In finite element formulation and within each element,

$$
\mathbf{u}=\mathbf{N}_{m} \widetilde{\mathbf{q}}_{m} e^{i \theta t}, \phi=\mathbf{N}_{e} \widetilde{\mathbf{q}}_{e} e^{i \theta t}, \boldsymbol{\tau}=\mathbf{P}_{m} \widetilde{\boldsymbol{\beta}}_{m} e^{i \theta t}, \mathbf{D}=\mathbf{P}_{e} \widetilde{\boldsymbol{\beta}}_{e} e^{i \theta t}
$$

It is trivial to show that the elementwise variational functionals in Eqn.(20), Eqn.(22), Eqn.(26) and Eqn.(30) will take the following form :

$$
\Pi^{e}=e^{2 i \theta t} \sum_{e} \frac{1}{2}\left\{\begin{array}{l}
\widetilde{\mathbf{q}}_{m} \\
\widetilde{\mathbf{q}}_{e}
\end{array}\right\}^{T}\left(\mathbf{k}^{e}+\theta^{2}\left\langle\rho \mathbf{N}_{m}^{T} \mathbf{N}_{m}\right\rangle\right)\left\{\begin{array}{l}
\widetilde{\mathbf{q}}_{m} \\
\widetilde{\mathbf{q}}_{e}
\end{array}\right\}
$$

which is stationary w.r.t. the nodal amplitude d.o.f.s. A standard eigenvalue problem is resulted.

\section{INTERPOLATION AND SHAPE FUNCTIONS}

In this section, a number of three-dimensional eight-node piezoelectric elements will be developed. For the eight-node element as depicted in Fig.2, the interpolation function for the i-th node is :

$$
N_{i}=\frac{1}{8}\left(1+\xi_{i} \xi\right)\left(1+\eta_{i} \eta\right)\left(1+\zeta_{i} \zeta\right)
$$

where $\xi, \eta$ and $\zeta \in[-1,+1]$ are the natural coordinates. Here, quantities with subscripts denote their nodal counterparts. The coordinates, displacement and electric potential are interpolated as :

$$
\begin{aligned}
& x=\sum_{i=1}^{8} N_{i} x_{i}, y=\sum_{i=1}^{8} N_{i} y_{i}, z=\sum_{i=1}^{8} N_{i} z_{i}, \phi=\left[N_{1}, \ldots, N_{8}\right]\left\{\phi_{1}, \ldots, \phi_{8}\right\}^{T}=\mathbf{N}_{e} \mathbf{q}_{e} \\
& \mathbf{u}=\left[N_{1} \mathbf{I}_{3}, \ldots, N_{8} \mathbf{I}_{3}\right]\left\{u_{1}, v_{1}, w_{1}, \ldots, u_{8}, v_{8}, w_{8}\right\}^{T}=\mathbf{N}_{m} \mathbf{q}_{m}
\end{aligned}
$$

where $\mathbf{I}_{i}$ is the $\mathrm{i}$-th order identity matrix. The following geometric parameters are defined for subsequent use : 


$$
\mathbf{T}_{e}=\left[\begin{array}{lll}
a_{1} & b_{1} & c_{1} \\
a_{2} & b_{2} & c_{2} \\
a_{3} & b_{3} & c_{3}
\end{array}\right]=\left[\left.\begin{array}{lll}
\partial x / \partial \xi & \partial y / \partial \xi & \partial z / \partial \xi \\
\partial x / \partial \eta & \partial y / \partial \eta & \partial z / \partial \eta \\
\partial x / \partial \zeta & \partial y / \partial \zeta & \partial z / \partial \zeta
\end{array}\right|_{\xi=\eta=\zeta=0}\right.
$$

which can be worked out to be :

$$
\mathbf{T}_{e}=\left[\begin{array}{lll}
a_{1} & b_{1} & c_{1} \\
a_{2} & b_{2} & c_{2} \\
a_{3} & b_{3} & c_{3}
\end{array}\right]=\frac{1}{8}\left[\begin{array}{cccccccc}
-1 & +1 & +1 & -1 & -1 & +1 & +1 & -1 \\
-1 & -1 & +1 & +1 & -1 & -1 & +1 & +1 \\
-1 & -1 & -1 & -1 & +1 & +1 & +1 & +1
\end{array}\right]\left\{\begin{array}{ccc}
x_{1} & y_{1} & z_{1} \\
\vdots & \vdots & \vdots \\
x_{8} & y_{8} & z_{8}
\end{array}\right\}
$$

It has been well-known that the element based solely on the above displacement interpolation is too stiff, susceptible to mesh distortion and aspect ratio. Ha, Keilers \& Chang [16] and Tzou [17] have supplemented the interpolated displacement with Wilson's incompatible modes [24,25] :

$$
\mathbf{u}=\mathbf{N}_{m} \mathbf{q}_{m}+\left[\left(1-\xi^{2}\right) \mathbf{I}_{3},\left(1-\eta^{2}\right) \mathbf{I}_{3},\left(1-\zeta^{2}\right) \mathbf{I}_{3}\right]\left\{\begin{array}{c}
\lambda_{1} \\
\vdots \\
\lambda_{9}
\end{array}\right\}
$$

It should be remarked that a modified strain-displacement operator suggested by Taylor, Beresford \& Wilson must be used [24]. Otherwise, the resulting element will fail the patch test. In finite element implementation, $\lambda_{i}$ 's are internal displacement d.o.f.s not shared by the adjacent elements. Hence, they can be condensed in the element level.

For the assumed stress, the one in Pian's element [34,37] are employed. The element contains eighteen stress modes which are minimal for securing the proper element rank. The stress in the element can be expressed as :

$$
\boldsymbol{\tau}=\mathbf{P}_{m} \boldsymbol{\beta}_{m}=\left[\begin{array}{ll}
\mathbf{I}_{6} & \mathbf{T}_{m} \mathbf{P}_{m}
\end{array}\right]\left\{\begin{array}{l}
\boldsymbol{\beta}_{m c} \\
\boldsymbol{\beta}_{m h}
\end{array}\right\}
$$

in which

$$
\mathbf{P}_{m h}=\left[\begin{array}{cccccccccccc}
0 & 0 & 0 & \eta & 0 & 0 & \zeta & 0 & 0 & \eta \zeta & 0 & 0 \\
\xi & 0 & 0 & 0 & 0 & 0 & 0 & \zeta & 0 & 0 & \zeta \xi & 0 \\
0 & \xi & 0 & 0 & \eta & 0 & 0 & 0 & 0 & 0 & 0 & \xi \eta \\
0 & 0 & 0 & 0 & 0 & 0 & 0 & 0 & \zeta & 0 & 0 & 0 \\
0 & 0 & \xi & 0 & 0 & 0 & 0 & 0 & 0 & 0 & 0 & 0 \\
0 & 0 & 0 & 0 & 0 & \eta & 0 & 0 & 0 & 0 & 0 & 0
\end{array}\right]
$$

is the higher order contravariant stress shape function matrix and 


$$
\mathbf{T}_{m}=\left[\begin{array}{cccccc}
a_{1}^{2} & a_{2}^{2} & a_{3}^{2} & 2 a_{1} a_{2} & 2 a_{2} a_{3} & 2 a_{3} a_{1} \\
b_{1}^{2} & b_{2}^{2} & b_{3}^{2} & 2 b_{1} b_{2} & 2 b_{2} b_{3} & 2 b_{3} b_{1} \\
c_{1}^{2} & c_{2}^{2} & c_{3}^{2} & 2 c_{1} c_{2} & 2 c_{2} c_{3} & 2 c_{3} c_{1} \\
a_{1} b_{1} & a_{2} b_{2} & a_{3} b_{3} & a_{1} b_{2}+a_{2} b_{1} & a_{2} b_{3}+a_{3} b_{2} & a_{3} b_{1}+a_{1} b_{3} \\
b_{1} c_{1} & b_{2} c_{2} & b_{3} c_{3} & b_{1} c_{2}+b_{2} c_{1} & b_{2} c_{3}+b_{3} c_{2} & b_{3} c_{1}+b_{1} c_{3} \\
c_{1} a_{1} & c_{2} a_{2} & c_{3} a_{3} & c_{1} a_{2}+c_{2} a_{1} & c_{2} a_{3}+c_{3} a_{2} & c_{3} a_{1}+c_{1} a_{3}
\end{array}\right]
$$

is the transformation matrix evaluated at the element origin for the contravariant and Cartesian stresses. It can be proven that the above stress is in strict homogenous equilibrium when the element Jacobian determinant is a constant.

For the electric displacement, the minimum number of assumed modes for securing the proper element rank is seven. To devise the electric displacement modes, a $2 \times 2 \times 2$ element with its natural and Cartesian coordinate axes parallel is considered. The interpolated electric potential can be expressed as :

$$
\phi=\left[\begin{array}{llllllll}
1 & \xi & \eta & \zeta & \xi \eta & \eta \zeta & \zeta \xi & \xi \eta \zeta
\end{array}\right]\left\{\begin{array}{c}
\psi_{1} \\
\vdots \\
\psi_{8}
\end{array}\right\}
$$

where $\psi_{i}$ 's are linear combinations of the element nodal electrical potential. The derived electric field is :

$$
\mathbf{E}=\left\{\begin{array}{l}
E_{\xi} \\
E_{\xi} \\
E_{\xi}
\end{array}\right\}=-\left\{\begin{array}{l}
\partial / \partial \xi \\
\partial / \partial \eta \\
\partial / \partial \zeta
\end{array}\right\} \phi=-\left[\begin{array}{cccccccc}
0 & 1 & 0 & 0 & \eta & 0 & \zeta & \eta \zeta \\
0 & 0 & 1 & 0 & \xi & \zeta & 0 & \zeta \xi \\
0 & 0 & 0 & 1 & 0 & \eta & \xi & \xi \eta
\end{array}\right]\left\{\begin{array}{c}
\psi_{1} \\
\vdots \\
\psi_{8}
\end{array}\right\}
$$

Recalling that -E and D are energy conjugates, the four non-constant or higher order electric field can be suppressed or matched by the following contravariant electric displacement modes :

$$
\left\{\begin{array}{c}
D_{\xi} \\
D_{\eta} \\
D_{\zeta}
\end{array}\right\}=\mathbf{P}_{e h} \boldsymbol{\beta}_{e h}=\left[\begin{array}{cccc}
\eta & 0 & \zeta & \eta \zeta \\
\xi & \zeta & 0 & \zeta \xi \\
0 & \eta & \xi & \xi \eta
\end{array}\right] \boldsymbol{\beta}_{e h}
$$

For a generic element, the assumed higher order Cartesian electric displacement can be transformed from $\mathbf{P}_{e h} \boldsymbol{\beta}_{e h}$. With the constant electric displacement augmented, the complete assumed electric displacement is :

$$
\mathbf{D}=\mathbf{P}_{e} \boldsymbol{\beta}_{e}=\left[\begin{array}{ll}
\mathbf{I}_{3} & \mathbf{T}_{e} \mathbf{P}_{e h}
\end{array}\right]\left\{\begin{array}{l}
\boldsymbol{\beta}_{e c} \\
\boldsymbol{\beta}_{e h}
\end{array}\right\}
$$


where $\mathbf{T}_{e}$ as defined in Eqn.(39) is the transformation matrix for the contravariant and Cartesian electric displacements evaluated at the element origin. It can be shown that the above assumed electric displacement satisfies the charge conservation condition when the element Jacobian determinant is a constant.

\section{NUMERICAL EXAMPLES}

In this section, a number of benchmark problems are examined. Predictions of the following elements are included for comparisons:

$\mathrm{H} 8$ - the irreducible element based on $\Pi$, the displacement and electric potential are given in Eqn.(38).

H8I - the irreducible incompatible element based on $\Pi$, the electric potential and displacement are given in Eqn.(38) and Eqn.(40), respectively.

H8D - the hybrid element based on $\Pi_{D}$, electric displacement is given in Eqn.(45) whereas displacement and electric potential are given in Eqn.(38).

H8DI - the hybrid incompatible element based on $\Pi_{D}$, the electric potential, displacement and electric displacement are given in Eqn.(38), Eqn.(40) and Eqn.(45), respectively.

H8S - the hybrid element based on $\Pi_{\tau}$, stress is given in Eqn.(41) whereas displacement and electric potential are given in Eqn.(38).

H8DS - the hybrid element based on $\Pi_{D \tau}$, the stress is given in Eqn.(41), electric displacement is given in Eqn.(45) whereas displacement and electric potential are given in Eqn.(38).

In the element abbreviations, "H", "8", "I", "D", "S" stand for hexahedron, eight-node, incompatible displacement, assumed electric displacement and assumed stress, respectively. All elements are evaluated by the second order Gaussian rule which is sufficient to secure the proper element rank.

Bimorph Beam - The bimorph beam is presented in the text of Tzou [17]. It consists of two identical PVDF uniaxial layers with opposite polarities and, hence, will bend when an electric field is applied in the transverse direction. Properties of PVDF are extracted from reference [17] and listed in Table 1. The bimorph is here modelled by eight elements at four elements per layer as depicted in Fig.3. With a unit voltage applied across the thickness, the free end deflection and normalized bending stress at the Gaussian point "A" closest to the top face are computed. The effect of mesh distortion on the element accuracy is examined by varying "e". The results are shown in Fig.4 and Fig.5. It can be seen that H8S/H8DS are better than H8I/H8DI whereas H8/H8D 
are extremely poor even at $\mathrm{e}=0$. All these elements are very sensitive to mesh distortion as a result of shear locking. Using a selective scaling technique which was developed for alleviated shear locking in hybrid stress solid elements [37], H8S/H8DS yield much better predictions as denoted by H8S*/H8DS* in the figures. H8DS* is marginally more accurate than H8S*.

Cantilever Beam - The problem depicted in Fig.6 was considered by Saravanos \& Heyliger [45]. The cantilever consists of a thick layer of unidirectional graphite/epoxy and a thin layer of PZT-4 piezoceramic adhered together. The fibre is running along the longitudinal direction of the beam. The material properties are listed in Table 1 . The beam is modelled with a total of $5 \times 8$ elements. The piezoelectric layer is modelled by a layer of 8 elements whereas the graphite/epoxy is modelled by 4 layers of 8 elements. A $12.5 \mathrm{kV}$ potential difference is applied across the piezoelectric layer. The computed deflection curve is shown in Fig.7. In obtaining the prediction from ABAQUS [46] for comparison, the cantilever is modelled by a total of $3 \times 16$ twenty-node hexahedral piezoelectric elements with one element layer for PZT-4 and two element layers for graphite/epoxy. As ABAQUS does not have an eight-node piezoelectric element, the twenty-node hexahedral element with designation C3D20E is selected [46]. The element is irreducible and fully integrated by the third order quadrature. In Fig.7, the results of Koko, Orisamolu, Smith \& Akpan [20] were calculated by $2 \times 8$ elements twenty-node composite elements. Predictions of all the eight-node elements are in between those of Koko et al [20] and ABAQUS. As the beam is quite thick, even H8/H8D can yield accurate results.

With graphite/epoxy replaced by aluminum (see Table 1 for material properties), eigenfrequencies of the structure is computed. Two circuit arrangements are considered. The first is an open circuit in which the bottom surface of the PZT-4 layer is grounded. The second is a closed circuit in which the top and bottom surfaces of the PZT-4 layer are both grounded. The ten lowest eigen frequencies are presented in Table 2 and Table 3. H8/H8D are most stiff as the predicted frequencies are much higher than that by H8I/H8DI, and H8S/H8DS. The tables also list the predictions given by Koko et al [20] and evaluated by ABAQUS. All the results are based on the same meshes described in the previous paragraph.

It can be seen in Table 2 and Table 3 that the first six frequencies predicted by H8I/H8DI and H8S/H8DS are in good agreement with that of Koko et al and ABAQUS. The differences are in the order of $0.5 \%$. When the beam is modelled by denser meshes, the difference in the higher order frequencies are reduced. For instance, the seventh and tenth frequencies predicted by $5 \times 16$ H8I/H8DI elements are $8803 \mathrm{~Hz}$ and $16095 \mathrm{~Hz}$ whereas the same frequencies predicted by the same 
number of H8S/H8DS elements are $8781 \mathrm{~Hz}$ and $16045 \mathrm{~Hz}$ under the open circuit arrangement. The dynamic predictions of H8S/H8DS are slightly more accurate than that of H8I/H8DI.

Simply Supported Laminated Square Plate with Bonded PZT-4 Layers - The problem portrayed in Fig.8 was first considered by Saravanos and Heyliger[22]. The structure is a simply supported square plate made of T300/934 graphite/epoxy with lay up [0/90/0]. Two layers of the PZT-4 are bonded to the top and bottom surface of the plate. The material properties have been given in Table 1. The length of the plate $L$ is $0.4 \mathrm{~m}$ and its total thickness $h$ is $0.008 \mathrm{~m}$. The surfaces of the PZT- 4 layers in contact with the graphite/epoxy laminate are grounded. Owing to symmetry, only the lower left hand quadrant of the structure is analysed. Using one layer of elements for each of the lamina and PZT-layer, $5 \times 4 \times 4$ and $5 \times 8 \times 8$ eight-node elements are employed for an eigen-frequency analysis. For comparison, the problem is also attempted by ABAQUS with $5 \times 8 \times 8 \mathrm{C} 3 \mathrm{D} 20 \mathrm{E}$ twentynode elements. The ten lowest computed frequencies are listed in Table 4. It can be noted that H8S/H8DS are more accurate than H8I/H8DI, especially for the higher frequencies using the coarse mesh. Again, H8DS marginally more accurate than H8S.

Simply Supported Laminated Square Plate with Boned PVDF Layers - This problem has been considered by Saravanos, Heyliger \& Ramirez [13], see Fig.8. It consists of three graphite/epoxy laminae plied at [90/0/90] and two PVDF layers bonded to the top and bottom surfaces. The material properties are listed in Table 5. The total thickness $h$ is $0.01 \mathrm{~m}$ and the length to thickness ratio, $L / h$, is 4 . Two load cases are considered. In the first one, a double-sinusoidal electric potential given as :

$$
\bar{\phi}=\sin \frac{\pi x}{L} \sin \frac{\pi y}{L}
$$

is applied to the top surface of the structure whereas the bottom surface and all the vertical edges are grounded. In the second case, a double-sinusoidal load :

$$
\bar{t}_{z}=\sin \frac{\pi x}{L} \sin \frac{\pi y}{L}
$$

is applied to the top surface of the structure whereas all the vertical edges, top and bottom surfaces are grounded. Same as the previous example, only one-quarter of the structure needs to be analysed. Three element layers are used to model each PVDF layer and two element layers are used to model each lamina. Hence, a total of twelve element layers are employed in the thickness direction. In constant z-plane, a $4 \times 4$ mesh is used. To obtain the stress and electric displacement along AA' and 
BB', their values at the second order quadrature points are extrapolated to the mid-points, which are optimal for linear elements, of the element edges coincident with AA' and BB'.

Under the double-sinusoidal electric potential, $\tau_{x x}$ along AA' and $\tau_{y z}$ along BB' are plotted in Fig.9 and Fig.10, respectively. H8I/H8DI and H8S/H8DS are all in good agreement with the exact solutions whereas as the elements with independently assumed electric displacement, i.e. H8DI and H8DS, are marginally more accurate than their counterparts without assumed electric displacement, i.e. H8I and H8S, respectively. The effect of mesh distortion on the central deflection is studied by varying the length "e" in Fig.11, the results are shown in Fig.12. It is seen that the assumed electric displacement can improves the element accuracy. The most accurate element is H8DS.

Under the double-sinusoidal mechanical load, $\tau_{x x}$ along AA', shear stress $\tau_{y z}$ along BB', electric potential $\phi$ along $\mathrm{AA}^{\prime}$ and electric displacement $D_{z}$ along $\mathrm{AA}^{\prime}$ are plotted in Fig.13 to Fig.16. In Fig.13 and Fig.14, the predictions using five element layers (one for each of the PVDF layer and graphite/epoxy lamina) are also obtained. All elements yield accurate $\tau_{x x}, \tau_{y z}$ and $\phi$. For $D_{z}$ shown in Fig.16, all elements yield accurate results in the graphite/epoxy laminate. The ones with assumed electric displacements are the better performers in the PVDF layers. The observation that H8DI and H8I are more accurate respectively than H8DS and H8S in the PVDF layers is due to the better fulfillment of the mechanical boundary conditions in H8DI and H8I as a result of the enforcement by the incompatible displacement modes, see Eqn.(6). Moreover, the incompatible modes provide a linear thickness variation of the transverse normal stress whereas the assumed transverse normal stress modes in H8S and H8DS are constant w.r.t. the thickness coordinate.

The effect of mesh distortion on the predicted central deflection can be seen in Fig.17. The assumed stress elements are more accurate than the incompatible elements.

\section{CLOSURE}

For piezoelectricity, the irreducible formulation is the one employing independently assumed displacement and electric potential. In this paper, hybrid eight-node hexahedral finite element models are formulated by employing variational functionals with assumed electric displacement, assumed stress and both. Comparing with the irreducible elements, the present hybrid elements are found to be more accurate as well as less sensitive to element distortion and aspect ratio.

Acknowledgment - The work described in this paper was substantially supported by a grant from the Research Grant Council of the Hong Kong SAR, P.R.China (Project No. HKU7082/97E). 
1. H.F.Tiersten, Linear piezoelectric Plate Vibrations, Plenum Press, New York, 1969

2. M.C.Ray, K.M.Rao and B.Samanta, 'Exact solution for static analysis of an intelligent structure under cylindrical bending', Computers \& Structures, 47, 1031-1042 (1993)

3. J.S.Yang, R.C.Batra, X.Q.Liang, "The cylindrical bending vibration of a laminated elastic plate due to piezoelectric actuators", Smart Mater. Struct., 3, 485-493 (1994)

4. P.R.Heyliger, D.A.Saravanos, "Exact free-vibration analysis of laminated piezoelectric layers", J.Acoustical Society of America, 98, 1547-1557 (1995)

5. A.Allik and T.J.R.Hughes, 'Finite element method for piezoelectric vibration', Inter.J.Numer. Methods Engrg., 2, 151-157 (1970)

6. M.Naillon, R.Coursant and F.Besnier, 'Analysis of piezoelectric structures by a finite element method', Acta Electronica, 25, 341-362 (1983)

7. J.H.Jeng, X.Bao, V.V.Varadan and V.K.Varadan, 'Complete finite element-eigenmode analysis for a 1-3 type of piezoelectric composite transducer including the effect of fluid loading and internal losses', Proc. IEEE 1988 Ultrasonics Symp., 685-688 (1988)

8. R.Lerch, 'Simulation of piezoelectric devices by two- and three-dimensional finite elements', IEEE Trans. Ultrasonics, Ferroelectrics \& Frequency Control, 37, 233-247 (1990)

9. J.A.Hossack and G.Hayward, 'Finite-element analysis of 1-3 composite transducers', IEEE Trans. Ultrasonics, Ferroelectrics \& Frequency Control, 38, 618-627 (1991)

10. N.-Q.Guo, P.Cawley and D.Hitchings, 'The finite element analysis of the vibration characteristics of piezoelectric disks', J.Sound \& Vibration, 159, 115-138 (1992)

11. W.-S.Hwang and H.C.Park, 'Finite element modeling of piezoelectric sensors and actuators', AIAA J., 31, 930-937 (1993)

12. M.A.Moetakef, K.L.Lawerence, S.P.Joshi and P.S.Shiakolas, 'Closed form expressions for higher order electroelastic tetrahedral elements', AIAA J. 33, 136-142 (1995)

13. P.Heyliger, G.Ramirez and D.Saravanos, 'Coupled discrete-layer finite elements for laminated piezoelectric plates', Commun. Numer.Methods Engrg., 10, 971-981 (1994)

14. H.S.Tzou and C.I.Tseng, 'Distributed piezoelectric sensor/actuator design for dynamic measurement/control of distributed parameter systems: a finite element approach', J.Sound \& Vibration, 138, 17-34 (1990)

15. R.Lammering, 'The application of a finite shell element for composites containing piezoelectric polymers in vibration control', Computers \& Structures, 41, 1101-1109 (1991)

16. S.K.Ha, C.Keilers and F.K.Chang, 'Finite element analysis of composite structures containing distributed piezoceramic sensors and actuators', AIAA J., 30, 772-780 (1992)

17. H.S.Tzou, Piezoelectric Shells: Distributed Sensing and Control of Continua, Kluwer Academic Publishers, Dordrecht, 1993

18. H.S.Tzou, C.I.Tseng and H.Bahrami, 'A thin piezoelectric hexaxedron finite element applied to design of smart continua', Finite Elements in Analysis \& Design, 16, 27-42, (1994)

19. H.S.Tzou, R.Ye, 'Analysis of piezoelectric structures with laminated piezoelectric triangle shell element', AIAA J., 34, 110-115 (1996)

20. T.S.Koko, I.R.Orisamolu, M.J.Smith and U.O.Akpan, 'Finite element based design tool for smart composite structures', Smart Structures and Materials 1997: Mathematics and Control in Smart Structures, ed. V.V.Varadan, J.Chandra, Proc. SPIE, 3039, 125-134 (1997)

21. J.Kim, V.V.Varadan and V.K.Varandan, 'Finite element modelling of structures including piezoelectric active devices', Inter.J.Numer.Methods Engrg., 40, 817-832 (1997)

22. D.A.Saravanos, P.R.Heyliger and D.H.Hopkins, 'Layerwise mechanics and finite element for the dynamic analysis of piezoelectric composite plates', Inter.J.Solids \& Structures, 34, 359378 (1997)

23. O.C.Zienkiewicz, R.L.Taylor, The Finite Element Method, Vol.1 - Basic Formulation and linear problems, $4^{\text {th }}$ Edn., McGraw-Hill, London, 1989

24. R.L.Taylor, P.J.Beresford and E.L.Wilson, 'A non-conforming element for stress analysis', Inter.J.Numer.Methods Engrg., 10, 1211-1220 (1976) 
25. J.C.Simo, M.S.Rifai, "A class of mixed assumed strain methods and the method of incompatible modes", Inter.J.Numer.Methods Engrg., 29, 1595-1638 (1990)

26. T.H.H.Pian, P.Tong, "Basis of finite elements for solids continua", Inter.J.Numer.Methods Engrg., 1, 3-28 (1969)

27. T.H.H.Pian, "Variational and finite element methods in structural analysis", RCA Review, 39, 648-664 (1978)

28. S.N.Atluri, P.Tong, H.Murakawa, "Recent studies in hybrid and mixed finite element methods in mechanics", in Hybrid and Mixed Finite Element Methods, S.N.Atluri, R.H.Gallagher \& O.C. Zienkiewicz (eds.), Chap.3, pp.51-71 (1983)

29. C.A.Felippa, "Parametrized multifield variational principles in elasticity : Part I. mixed functionals \& Part II. hybrid functionals", Comm.Appl.Numer. Methods, 5, 79-98 (1989)

30. T.H.H.Pian, "State-of-the-art Development of Hybrid/Mixed Finite Element Method", Finite Elements in Analysis \& Design, 21, 5-20 (1995)

31. T.H.H.Pian, S.W.Lee, "Notes of finite elements for nearly incompressible materials", AIAA J., 14, 824-826 (1976)

32. K.Washizu, Variational Methods in Elasticity and Plasticity, $3^{\text {rd }}$ Edition, Pergamon Press, Oxford, 1983

33. S.W.Lee, T.H.H.Pian, "Improvement of plate and shell finite elements by mixed formulations", AIAA.J., 16, 29-34 (1978)

34. T.H.H.Pian, "Finite elements based on consistently assumed stresses and displacements", Finite Elements in Analysis \& Design, 1, 131-140 (1985)

35. S.W.Lee, J.J.Rhiu, "A new efficient approach to the formulation of mixed finite element models for structural analysis", Inter.J.Numer.Methods Engrg., 21, 1629-1641 (1986)

36. C.-C.Wu, M.-G.Huang, T.H.H.Pian, "Consistency condition and convergence criteria of incompatible elements: general formulation of incompatible functions and its application", Computers \& Structures, 27, 639-644 (1987)

37. K.Y.Sze and A.Ghali, 'Hybrid hexahedral element for solids, plates, shells and beams by selective scaling', Inter.J.Numer.Methods Engrg., 36, 1519-1540 (1993)

38. K.Y.Sze, "An explicit hybrid-stabilized 9-node Lagrangian shell element", Computer Methods Appl.Mech. Engrg., 117, 361-379 (1994)

39. K.Y.Sze, "Stabilization schemes for 12-node to 21-node brick elements based on orthogonal and consistently assumed stress modes", Computer Methods App.Mech.Engrg., 119, 325-340 (1994)

40. K.Y.Sze, H.Fan, C.L.Chow, "Elimination of spurious kinematic and pressure modes in biquadratic plane element", Inter.J.Numer.Methods Engrg., 38, 3911-3932 (1995)

41. W.-J.Chen, Y.K.Cheung, "Non-conforming element method and refined hybrid method for axisymmetric solids", Inter.J.Numer.Methods Engrg., 39, 2509-2529 (1996)

42. K.Ghandi and N.W.Hagood, 'A hybrid finite element model for phase transitions in nonlinear electro-mechanically coupled material', Smart Structures and Materials 1997: Mathematics and Control in Smart Structures, ed. V.V.Varadan \& J.Chandra, Proc. SPIE, 3039, 97-112 (1997)

43. E.P.EerNisse, 'Variational method for electroelastic vibration analysis', IEEE Trans. Sonics and Ultrasonics, 14, 153-160 (1967)

44. J.S.Yang, 'Variational formulations for the vibration of a piezoelectric body', Quar.J.of Appl. Math., 53, 95-104 (1995)

45. D.A.Saravanos, P.R.Heyliger, "Coupled layerwise analysis of composite beams with embedded piezoelectric sensors and actuators", J.Intelligent Matl. Systems \& Struct., 6, 350-363 (1995)

46. ABAQUS Theory Manual, Version 5.6, Hibbitt, Karlsson \& Sorensen Inc., Rhode Island, 1997 
Table 1. Material properties

\begin{tabular}{|c|c|c|c|c|}
\hline & T300/934 Gr/Epoxy & PZT-4 & PVDF & $\mathrm{Al}$ \\
\hline \multicolumn{5}{|l|}{ Elastic Properties (in $\mathbf{c}_{E}$ ) } \\
\hline$E_{11}(\mathrm{GPa})$ & 132.8 & 83.0 & 2.0 & 68.9 \\
\hline$E_{22}(\mathrm{GPa})$ & 10.76 & 81.3 & 2.0 & 68.9 \\
\hline$E_{33}(\mathrm{GPa})$ & 10.96 & 66.0 & 2.0 & 68.9 \\
\hline$G_{12}=G_{13}(\mathrm{GPa})$ & 5.65 & 31.0 & & \\
\hline$G_{23}(\mathrm{GPa})$ & 3.61 & 25.6 & & \\
\hline$v_{12}=v_{13}$ & 0.24 & 0.31 & 0.29 & 0.25 \\
\hline$v_{23}$ & 0.49 & 0.43 & 0.29 & 0.25 \\
\hline \multicolumn{5}{|c|}{ Piezoelectric Coefficients (in matrix $\mathbf{d}$ or $\mathbf{e}$ ) } \\
\hline$d_{31}=d_{32}\left(10^{-12} \mathrm{~m} / \mathrm{V}\right)$ & & -122 & & \\
\hline$d_{33}\left(10^{-12} \mathrm{~m} / \mathrm{V}\right)$ & & 285 & & \\
\hline$e_{31}\left(\mathrm{C} / \mathrm{m}^{2}\right)$ & & & 0.046 & \\
\hline \multicolumn{5}{|c|}{ Electric Permittivity Coeffiicnets (in matrix $\mathrm{e}_{\gamma}$ ) } \\
\hline$\varepsilon_{11}=\varepsilon_{22}\left(10^{-8} \mathrm{Farad} / \mathrm{m}\right)$ & & 1.3054 & 0.01062 & \\
\hline$\varepsilon_{33}\left(10^{-8} \mathrm{Farad} / \mathrm{m}\right)$ & & 1.1505 & 0.01062 & \\
\hline Mass Density $\left(\mathrm{kg} / \mathrm{m}^{3}\right)$ & 1578 & 7600 & 1800 & 2769 \\
\hline
\end{tabular}

Table 2. Eigen frequencies $(\mathrm{Hz})$ of Al beam with a PZT-4 layer under open circuit, see Fig.6

\begin{tabular}{ccccccccc}
\hline $\begin{array}{c}\text { model } \\
\text { (no. of elements) }\end{array}$ & $\begin{array}{c}\text { H8I } \\
(5 \times 8)\end{array}$ & $\begin{array}{c}\text { H8 } \\
(5 \times 8)\end{array}$ & $\begin{array}{c}\text { H8S } \\
(5 \times 8)\end{array}$ & $\begin{array}{c}\text { H8ID } \\
(5 \times 8)\end{array}$ & $\begin{array}{c}\text { H8D } \\
(5 \times 8)\end{array}$ & $\begin{array}{c}\text { H8DS } \\
(5 \times 8)\end{array}$ & $\begin{array}{c}\text { Koko }[20] \\
(2 \times 8)\end{array}$ & $\begin{array}{c}\text { ABAQUS } \\
(3 \times 16)\end{array}$ \\
\hline 1 & 562.1 & 690.0 & 559.6 & 562.1 & 690.0 & 559.7 & 556.4 & 557.8 \\
2 & 819.5 & 934.4 & 815.9 & 819.6 & 934.4 & 815.7 & 818.3 & 820.3 \\
3 & 3447.9 & 4166.1 & 3433.3 & 3448.5 & 4166.1 & 3434.5 & 3307.6 & 3308.1 \\
4 & 4305.0 & 4313.2 & 4288.0 & 4305.0 & 4313.2 & 4288.0 & 4323.5 & 4262.2 \\
5 & 4807.4 & 5365.4 & 4789.4 & 4807.8 & 5365.4 & 4789.3 & 4651.6 & 4664.9 \\
6 & 7771.2 & 7789.3 & 7762.4 & 7771.4 & 7789.4 & 7763.1 & 7721.8 & 7736.7 \\
7 & 9503.0 & 11243 & 9455.0 & 9506.5 & 11243 & 9459.8 & 8629.0 & 8603.8 \\
8 & 12388 & 13030 & 12351 & 12389 & 13030 & 12352 & 11490 & 11485 \\
9 & 13252 & 13807 & 13166 & 13253 & 13807 & 13167 & 13047 & 12880 \\
10 & 18392 & 21259 & 18280 & 18403 & 21259 & 18293 & 15564 & 15428 \\
\hline
\end{tabular}

Table 3. Eigen frequencies (Hz) of Al beam with a PZT-4 layer under closed circuit, see Fig.6

\begin{tabular}{ccccccccc}
\hline $\begin{array}{c}\text { model } \\
\text { (no. of elements) }\end{array}$ & $\begin{array}{c}\text { H8I } \\
(5 \times 8)\end{array}$ & $\begin{array}{c}\text { H8 } \\
(5 \times 8)\end{array}$ & $\begin{array}{c}\text { H8S } \\
(5 \times 8)\end{array}$ & $\begin{array}{c}\text { H8ID } \\
(5 \times 8)\end{array}$ & $\begin{array}{c}\text { H8D } \\
(5 \times 8)\end{array}$ & $\begin{array}{c}\text { H8DS } \\
(5 \times 8)\end{array}$ & $\begin{array}{c}\text { Koko [20] } \\
(2 \times 8)\end{array}$ & $\begin{array}{c}\text { ABAQUS } \\
(3 \times 16)\end{array}$ \\
\hline 1 & 556.4 & 683.8 & 554.3 & 556.5 & 683.8 & 554.5 & 551.4 & 551.4 \\
2 & 816.7 & 928.4 & 812.5 & 816.7 & 928.4 & 812.6 & 817.2 & 816.4 \\
3 & 3417 & 4133 & 3404 & 3417 & 4133 & 3405 & 3280 & 3273 \\
4 & 4305 & 4313 & 4288 & 4305 & 4313 & 4288 & 4324 & 4262 \\
5 & 4794 & 5337 & 4773 & 4794 & 5337 & 4774 & 4646 & 4646 \\
6 & 7738 & 7752 & 7730 & 7739 & 7752 & 7731 & 7689 & 7699 \\
7 & 9428 & 11172 & 9389 & 9429 & 11172 & 9392 & 8573 & 8522 \\
8 & 12364 & 13022 & 12325 & 12364 & 13022 & 12325 & 11479 & 11449 \\
9 & 13247 & 13759 & 13158 & 13247 & 13759 & 13160 & 13046 & 12874 \\
10 & 18276 & 21160 & 18184 & 18278 & 21160 & 18192 & 15491 & 15297 \\
\hline
\end{tabular}


Table 4: Eigen frequencies $(\mathrm{Hz})$ of simply supported laminates with PZT-4 layers, see Fig.8

\begin{tabular}{cccccccccccc}
\hline $\begin{array}{c}\text { mode } \\
\text { no. }\end{array}$ & $\begin{array}{c}\text { H8 } \\
(4 \times 4)\end{array}$ & $\begin{array}{c}\text { H8I } \\
(4 \times 4)\end{array}$ & $\begin{array}{c}\text { H8S } \\
(4 \times 4)\end{array}$ & $\begin{array}{c}\text { H8DI } \\
(4 \times 4)\end{array}$ & $\begin{array}{c}\text { H8DS } \\
(4 \times 4)\end{array}$ & $\begin{array}{c}\text { H8 } \\
(8 \times 8)\end{array}$ & $\begin{array}{c}\text { H8I } \\
(8 \times 8)\end{array}$ & $\begin{array}{c}\text { H8S } \\
(8 \times 8)\end{array}$ & $\begin{array}{c}\text { H8DI } \\
(8 \times 8)\end{array}$ & $\begin{array}{c}\text { H8DS } \\
(8 \times 8)\end{array}$ & $\begin{array}{c}\text { ABAQUS } \\
(8 \times 8)\end{array}$ \\
\hline 1 & 439.0 & 239.2 & 235.6 & 239.2 & 235.5 & 296. & 232.6 & 232.4 & 232.6 & 232.4 & 231.4 \\
2 & 2731. & 1242. & 1205. & 1243. & 1204. & $\begin{array}{c}3 \\
1553\end{array}$ & 1064. & 1063. & 1065. & 1063. & 1024. \\
3 & 3071. & 1610. & 1578. & 1612. & 1577. & 1852 & 1396. & 1395. & 1396. & 1395. & 1342. \\
4 & 4216. & 2658. & 2317. & 2660. & 2306. & 2625 & 2075. & 2061. & 2076. & 2058. & 1984. \\
5 & 5975. & 4349. & 4212. & 4359. & 4209. & 4264 & 2866. & 2862. & 2868. & 2862. & 2568. \\
6 & 8138. & 5339. & 4930. & 5349. & 4905. & 4956 & 3721. & 3679. & 3724. & 3672. & 3358. \\
7 & 8634. & 5964. & 5209. & 5965. & 5209. & 5019 & 3726. & 3722. & 3729. & 3722. & 3382. \\
8 & 9276. & 6142. & 5680. & 6150. & 5665. & 5512 & 4304. & 4262. & 4307. & 4257. & 3900. \\
9 & 9571. & 6850. & 5964. & 6859. & 5963. & 5935 & 5710. & 5579. & 5714. & 5563. & 4738. \\
10 & 9660. & 8133. & 7388. & 8133. & 7347. & 7139 & 5817. & 5809. & 5824. & 5808. & 5083.
\end{tabular}

Table 5: Material Properties for the Piezoelectric Laminate

\begin{tabular}{ccc}
\hline & Graphite/epoxy & PVDF \\
\hline Elastic Coefficients (in matix $\left.\mathbf{c}_{E}\right)$ & & 238.0 \\
$c_{11}(\mathrm{GPa})$ & 134.9 & 23.6 \\
$c_{22}(\mathrm{GPa})$ & 14.35 & 10.6 \\
$c_{33}(\mathrm{GPa})$ & 14.35 & 3.98 \\
$c_{12}(\mathrm{GPa})$ & 5.156 & 2.19 \\
$c_{13}(\mathrm{GPa})$ & 5.156 & 1.92 \\
$c_{23}(\mathrm{Gpa})$ & 7.133 & 2.15 \\
$c_{44}(\mathrm{Gpa})$ & 3.606 & 4.40 \\
$c_{55}(\mathrm{Gpa})$ & 5.654 & 6.43 \\
$c_{66}(\mathrm{Gpa})$ & 5.654 & -0.13 \\
$e_{31}\left(\mathrm{C} / \mathrm{m}^{2}\right)$ & & -0.14 \\
$e_{32}\left(\mathrm{C} / \mathrm{m}^{2}\right)$ & & -0.28 \\
$e_{33}\left(\mathrm{C} / \mathrm{m}^{2}\right)$ & & -0.01 \\
$e_{25}=e_{16}\left(\mathrm{C} / \mathrm{m}^{2}\right)$ & & \\
\hline Piezoelectric Coefficints (in matrix $\mathbf{e})$ & & 12.50 \\
$\varepsilon_{11} / \varepsilon_{\mathrm{o}}$ & 3.5 & 11.98 \\
\hline$\varepsilon_{33} / \varepsilon_{0}=\varepsilon_{22} / \varepsilon_{0}$ & 3.0 & \\
\hline$\varepsilon_{0}($ permittivity of free space) & $8.854 \times 10^{-12}(\mathrm{Farad} / \mathrm{m})$ & \\
\hline
\end{tabular}



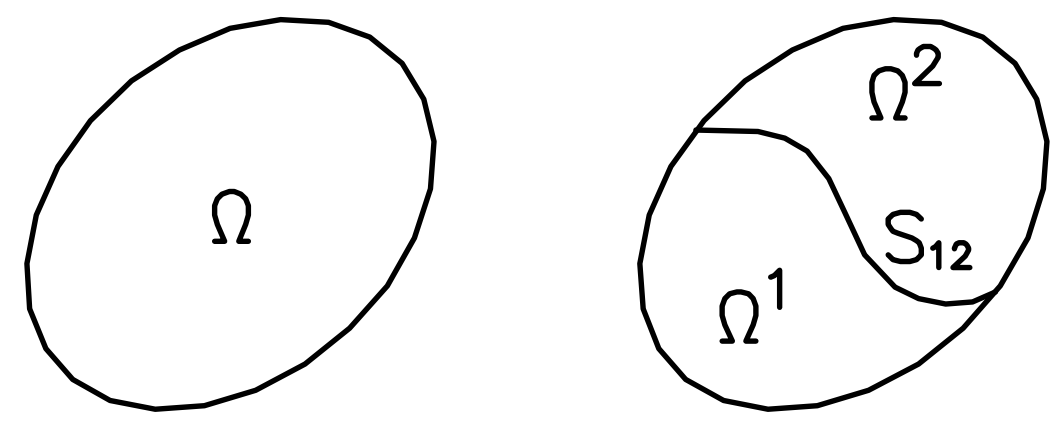

Fig.1. A piezoelectric domain $\Omega$ (left) and its sub-domains $\Omega^{1}$ and $\Omega^{2}$ (right), $\mathrm{S}_{12}$ is the sub-domain interface

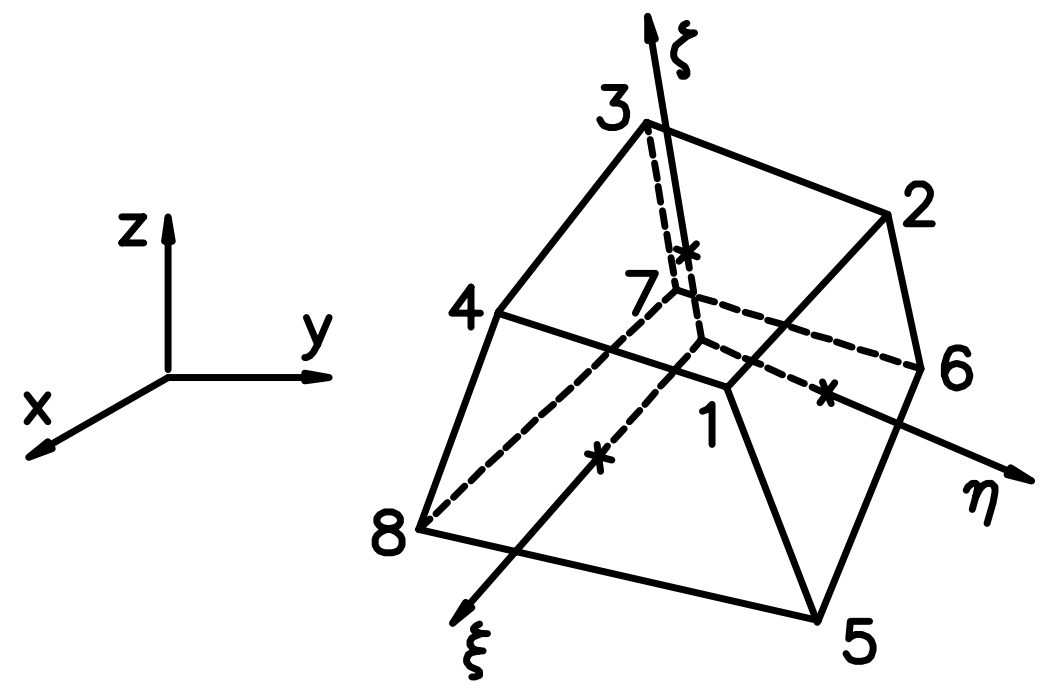

Fig.2. An eight-node hexahedral element and its node numbering sequence

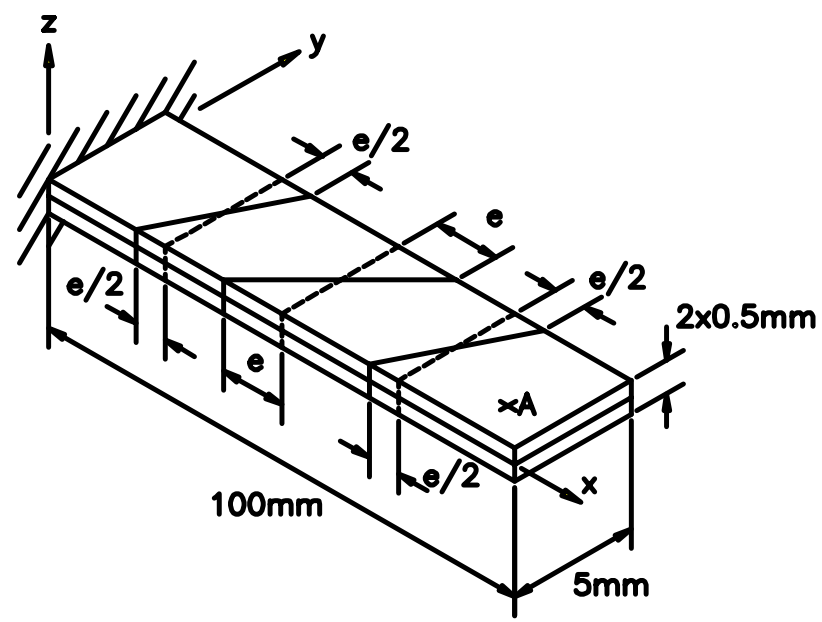

Fig.3. A bimorph cantilever 


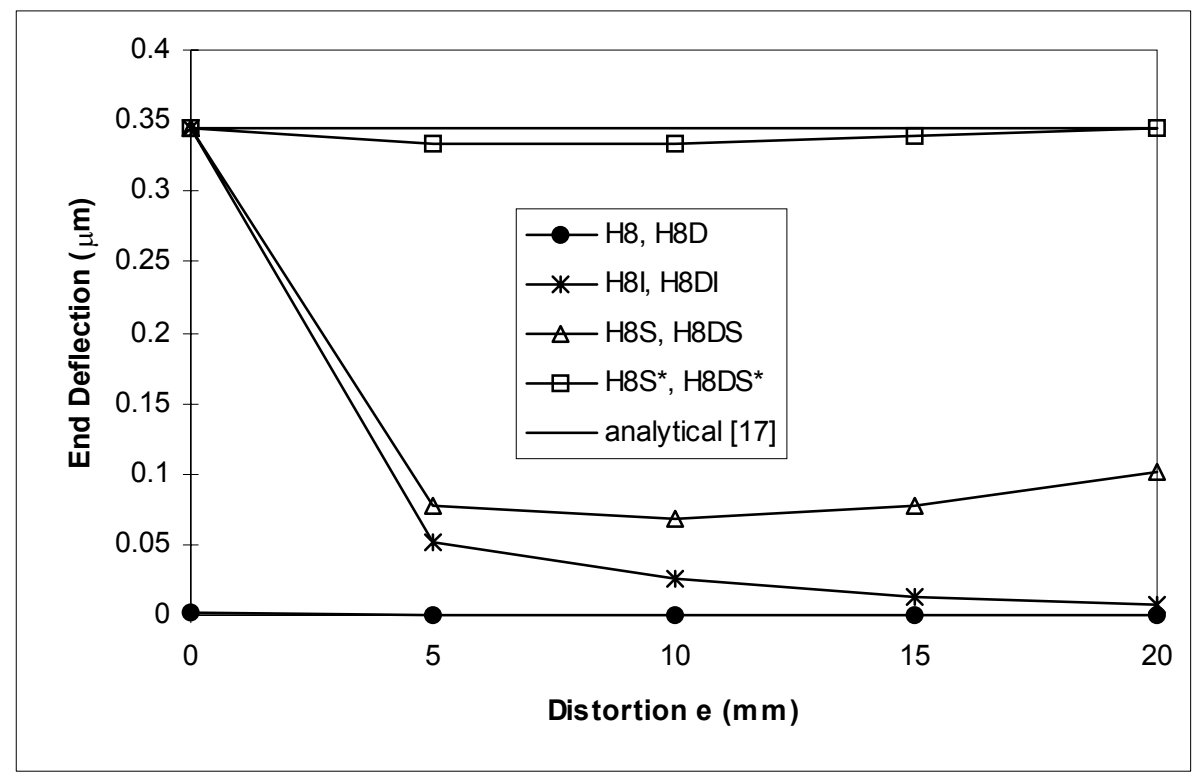

Fig.4. Effect of mesh distortion on the end deflection of the bimorph cantilever in Fig.3;

H8S* and H8DS* employ the selective scaling technique [37]

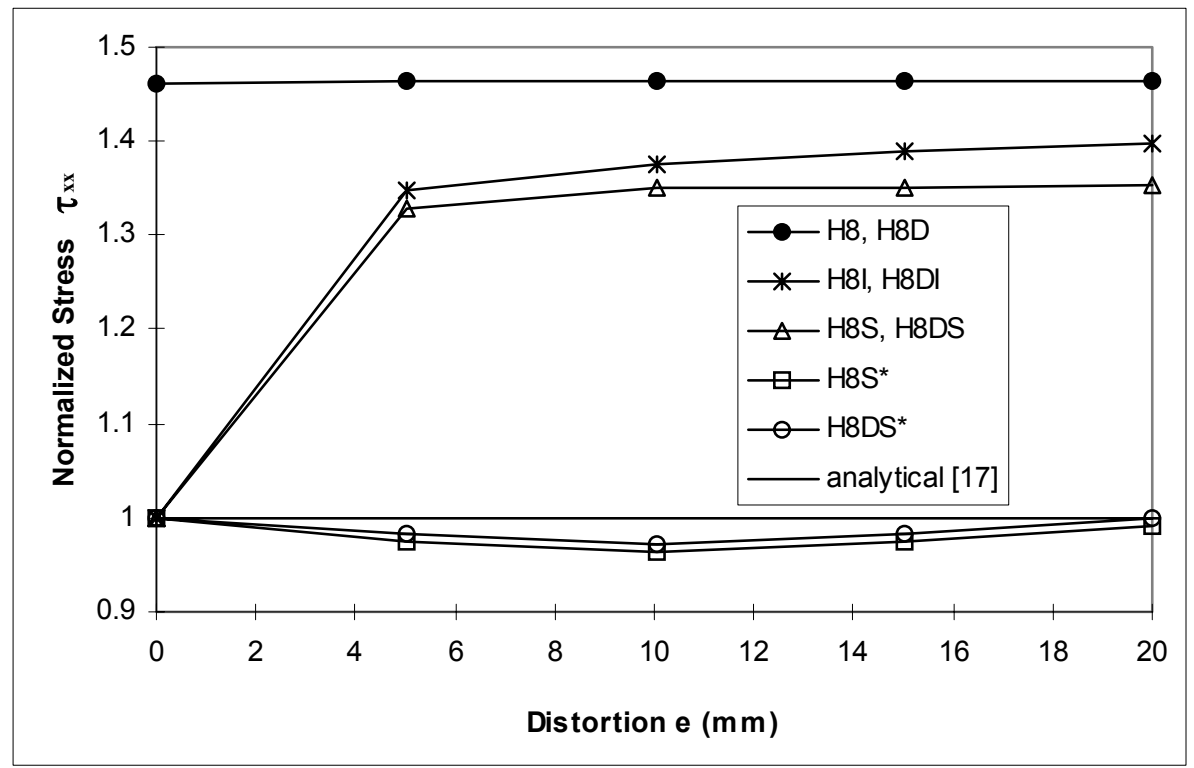

Fig.5. Effect of mesh distortion on the bending stress $\tau_{\mathrm{xx}}$ in the bimorph cantilever, see Fig.3; H8S* and H8DS* employ the selective scaling technique [37] 

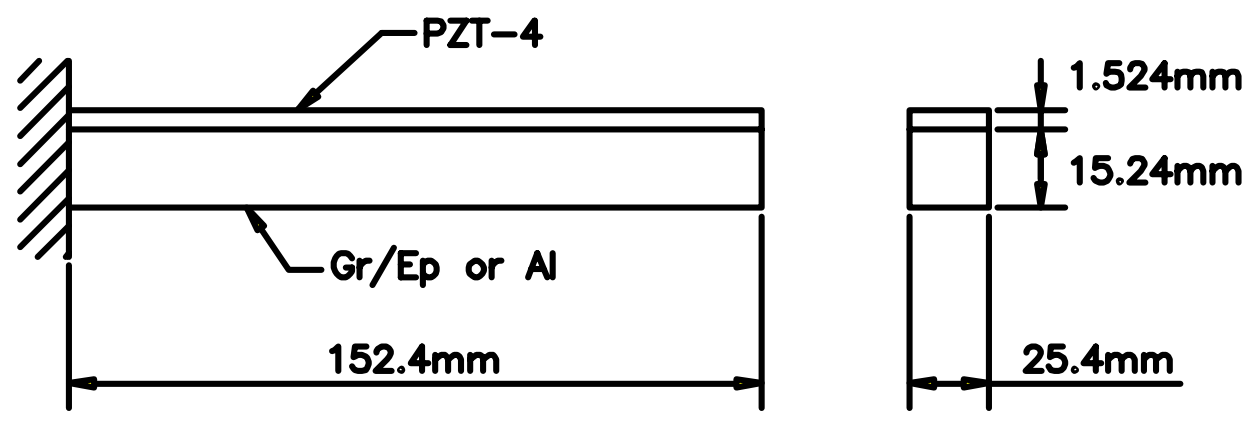

Fig.6. A cantilever with an adhered piezoelectric layer

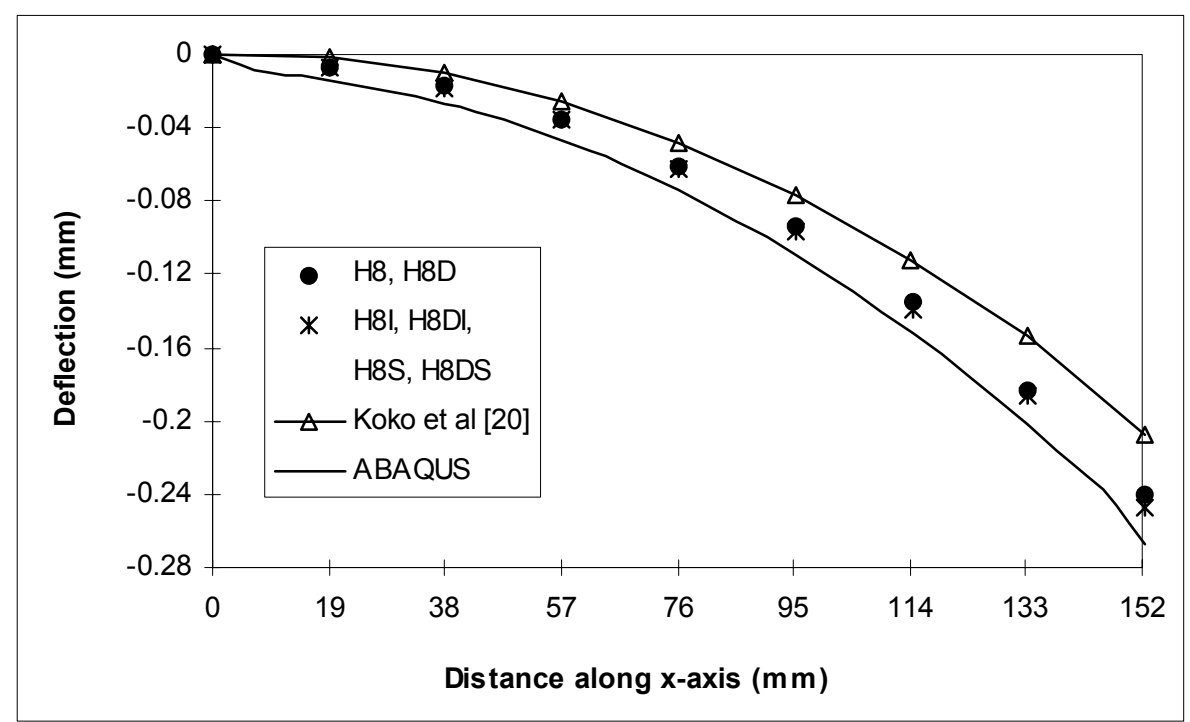

Fig.7. Deflection curve of the cantilever shown in Fig.6 under electric loading

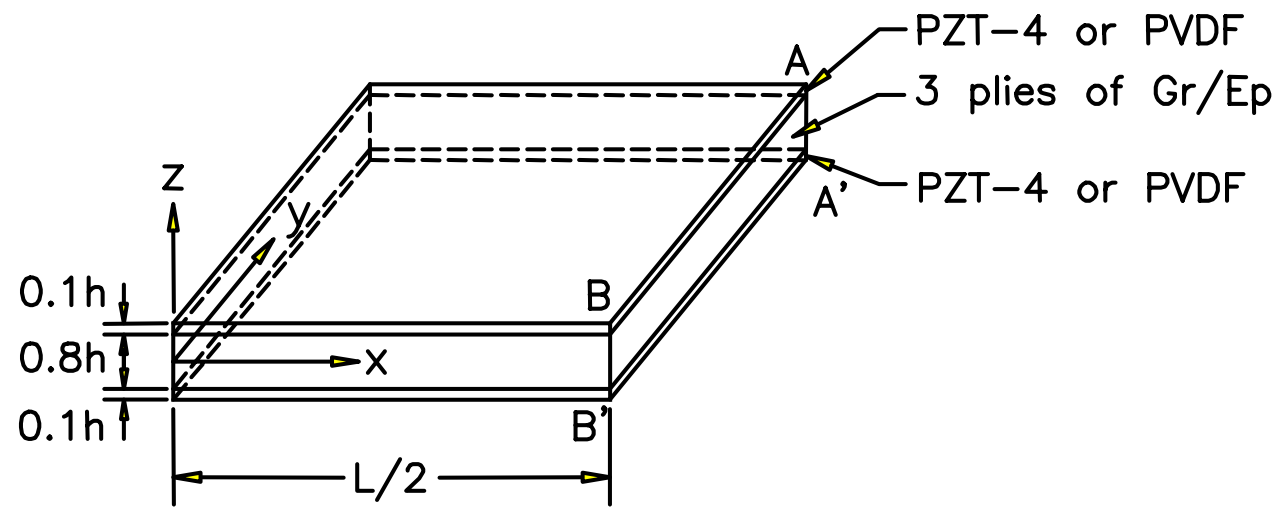

Fig.8. A quadrant of a simply supported three-ply composite plate with two adhered piezoelectric layers. AA' is the centre of the plate 


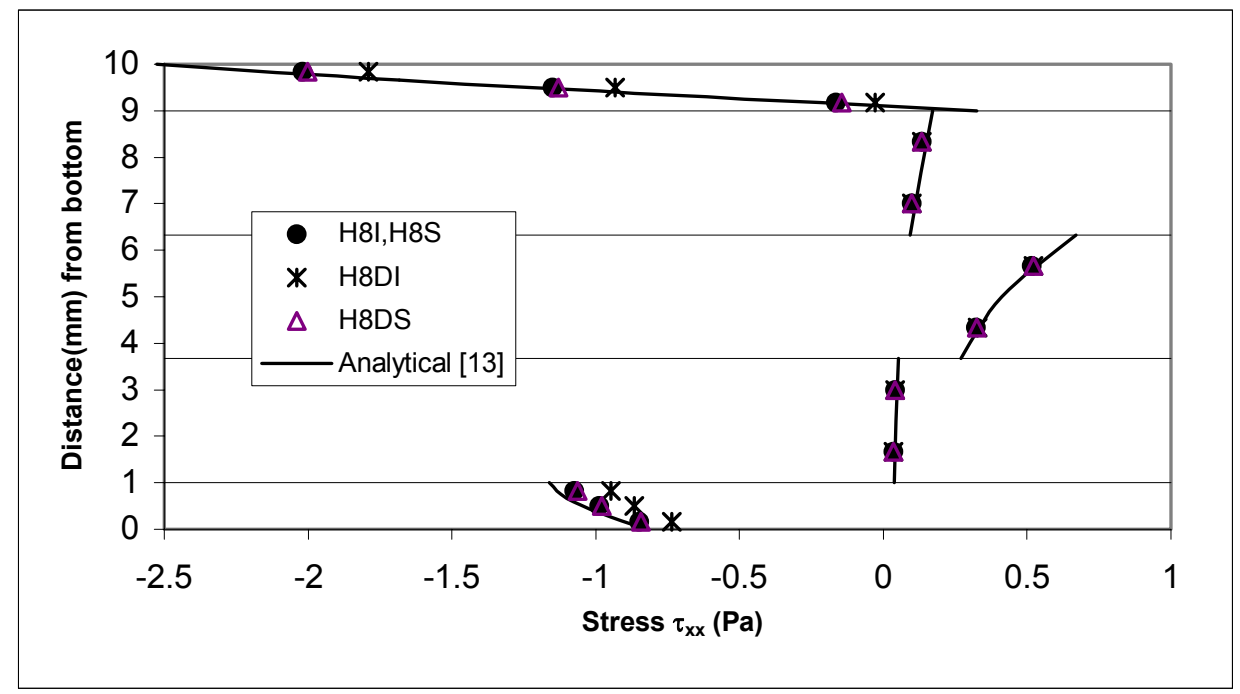

Fig.9. Variation of $\tau_{\mathrm{xx}}$ along AA' for the simply supported laminated plate under an applied double-sinusoidal electric potential, see Fig.8

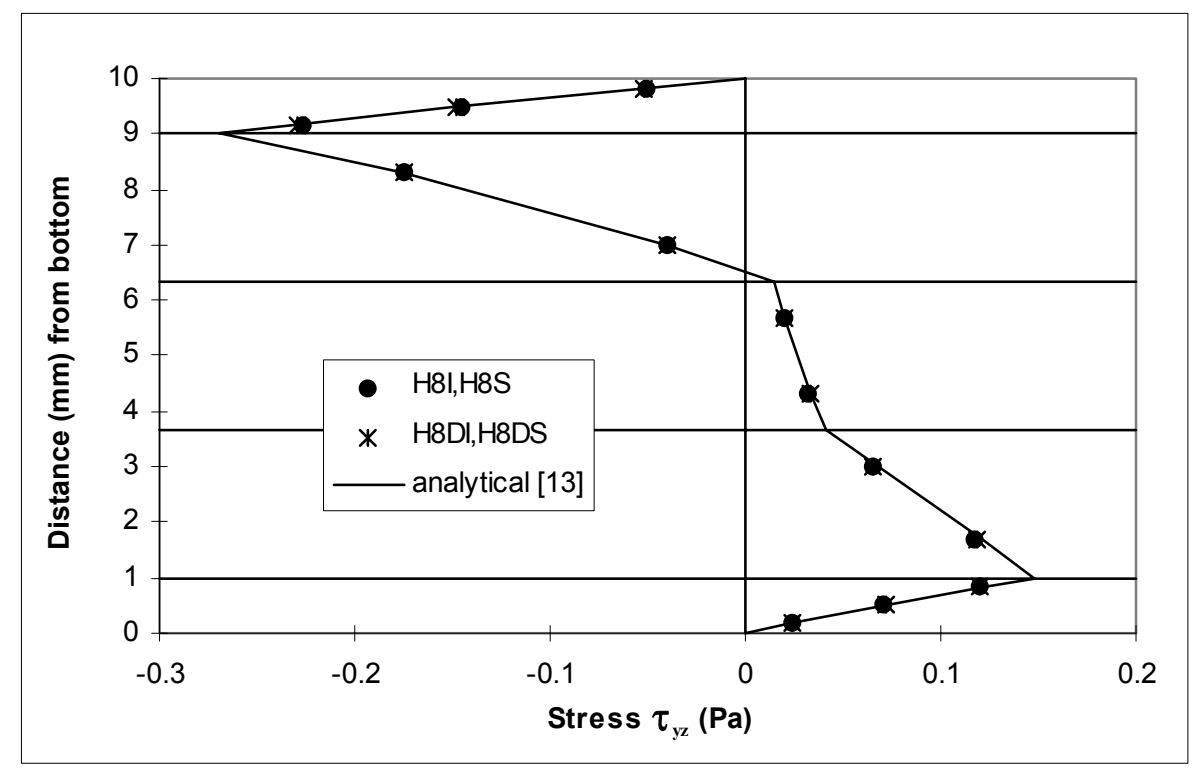

Fig.10. Variation of $\tau_{\mathrm{yz}}$ along BB' for the simply supported laminated plate under an applied double-sinusoidal electric potential, see Fig.8 


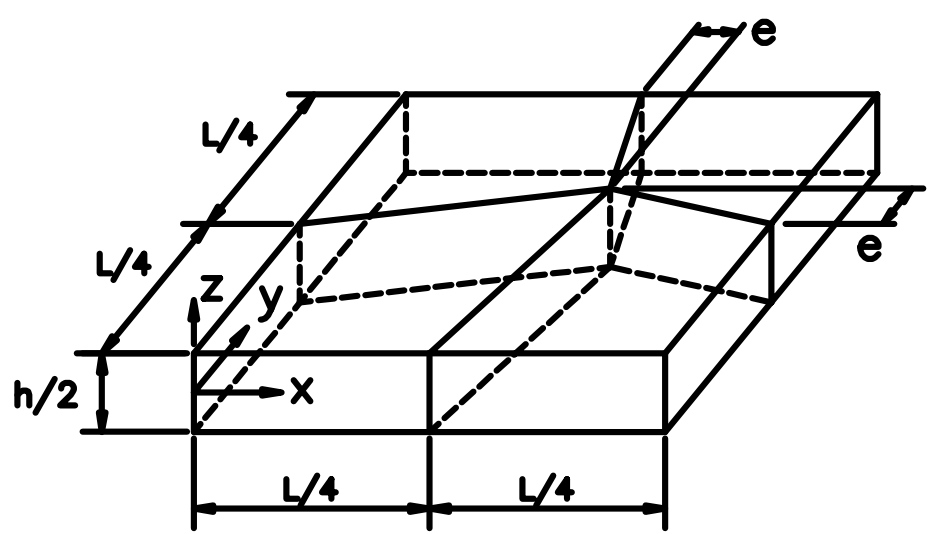

Fig.11. Distorted mesh for the lower left hand quadrant of the laminated plate, see Fig. 8

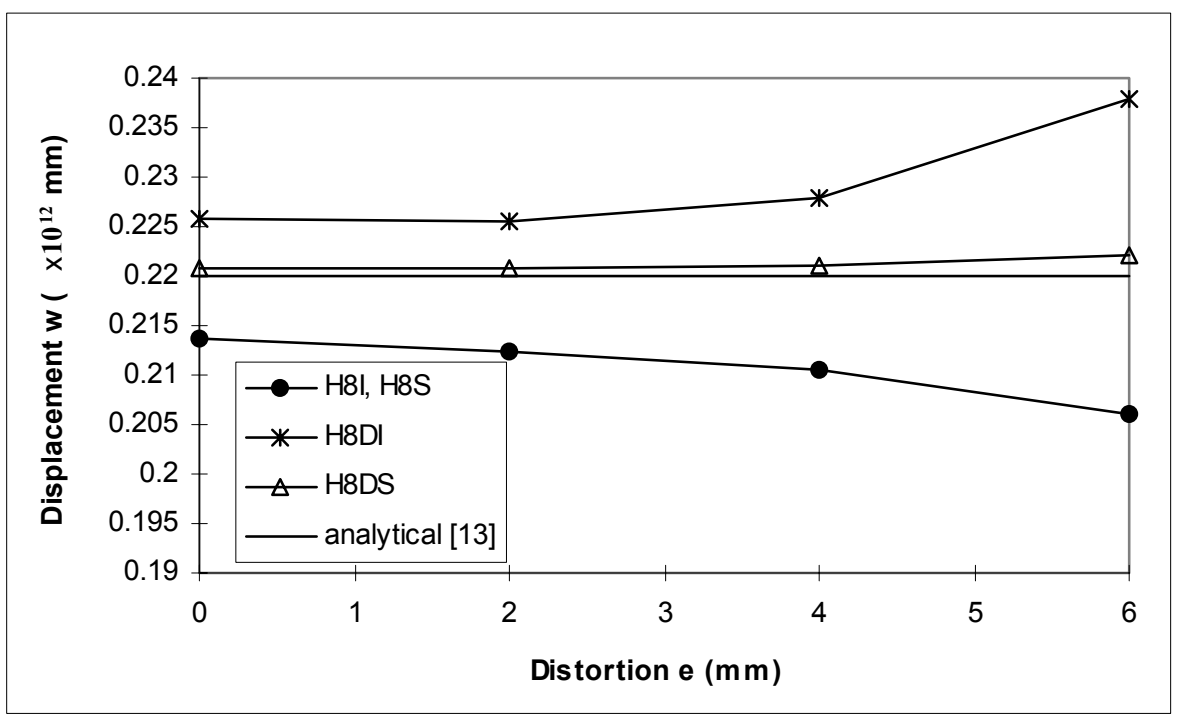

Fig.12. Effect of mesh distortion on the central vertical deflection of the simply supported laminated plate under an applied double-sinusoidal electric potential, see Fig. 11

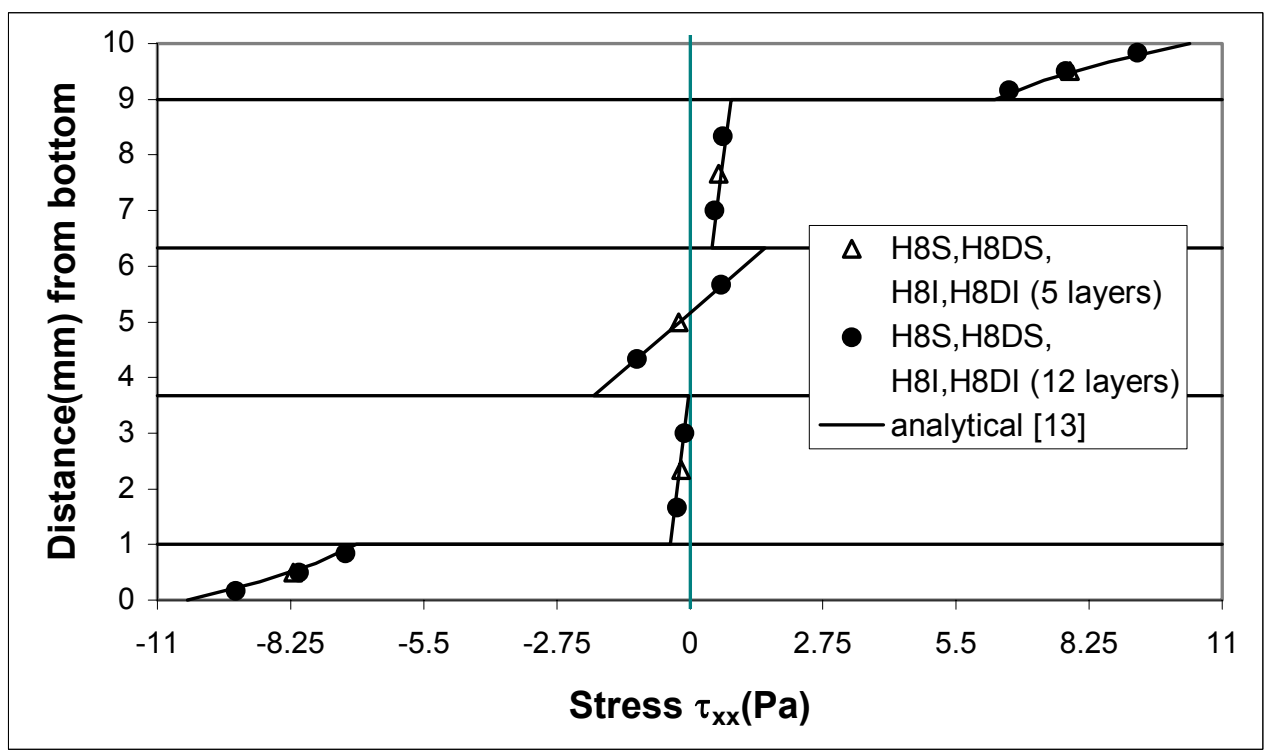

Fig.13. Variation of $\tau_{\mathrm{xx}}$ along AA' of the simply supported laminated plate under an applied double-sinusoidal mechanical load, see Fig. 11 


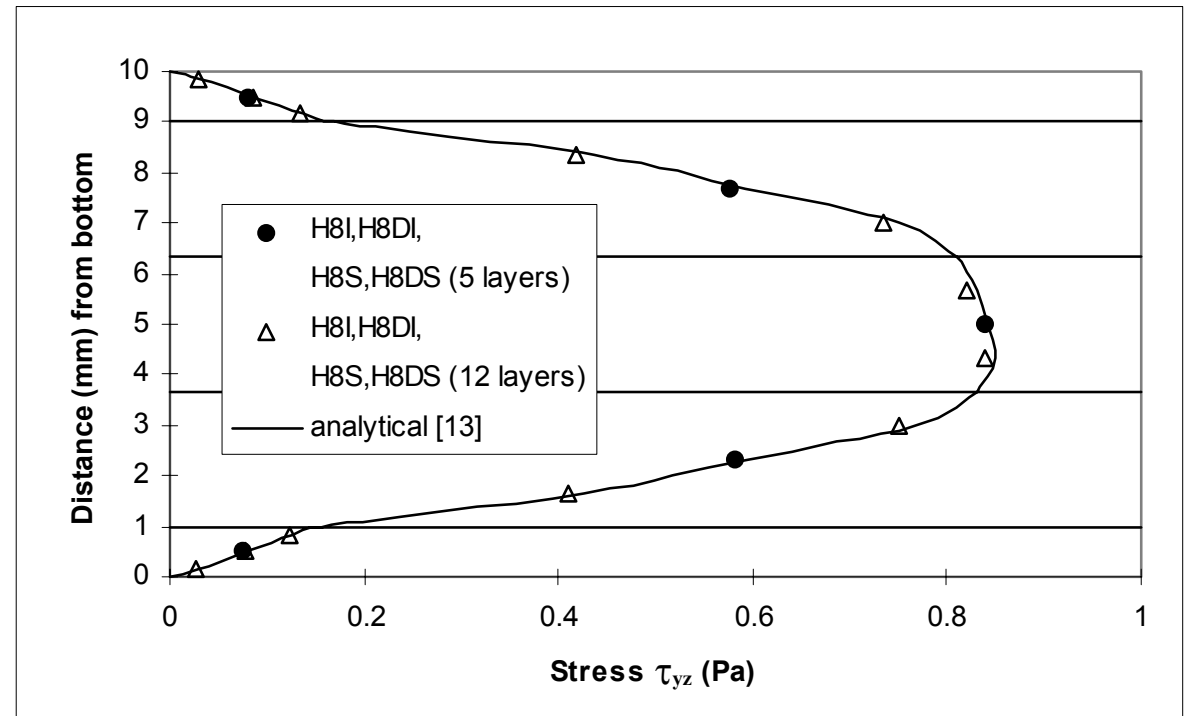

Fig.14. Variation of $\tau_{\mathrm{yz}}$ along BB' of the simply supported laminated plate under an applied double-sinusoidal mechanical load, see Fig.8

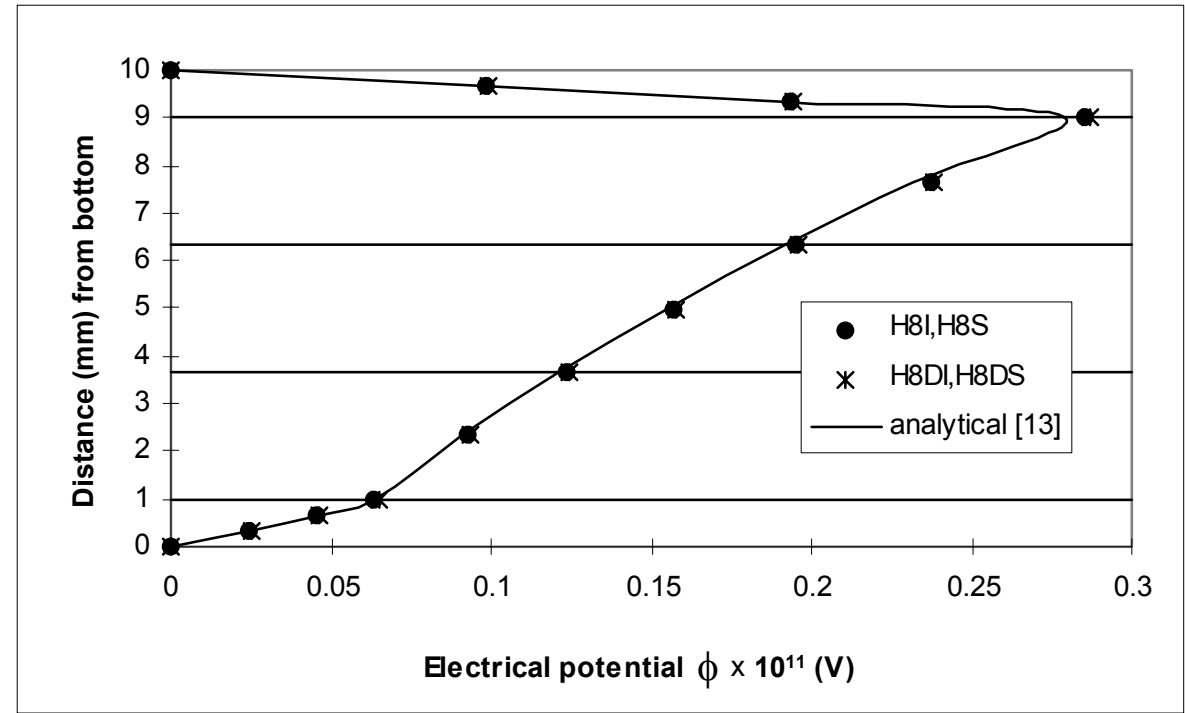

Fig.15. Variation of electric potential along AA' of the simply supported laminated plate under an applied double-sinusoidal mechanical load, see Fig. 8 


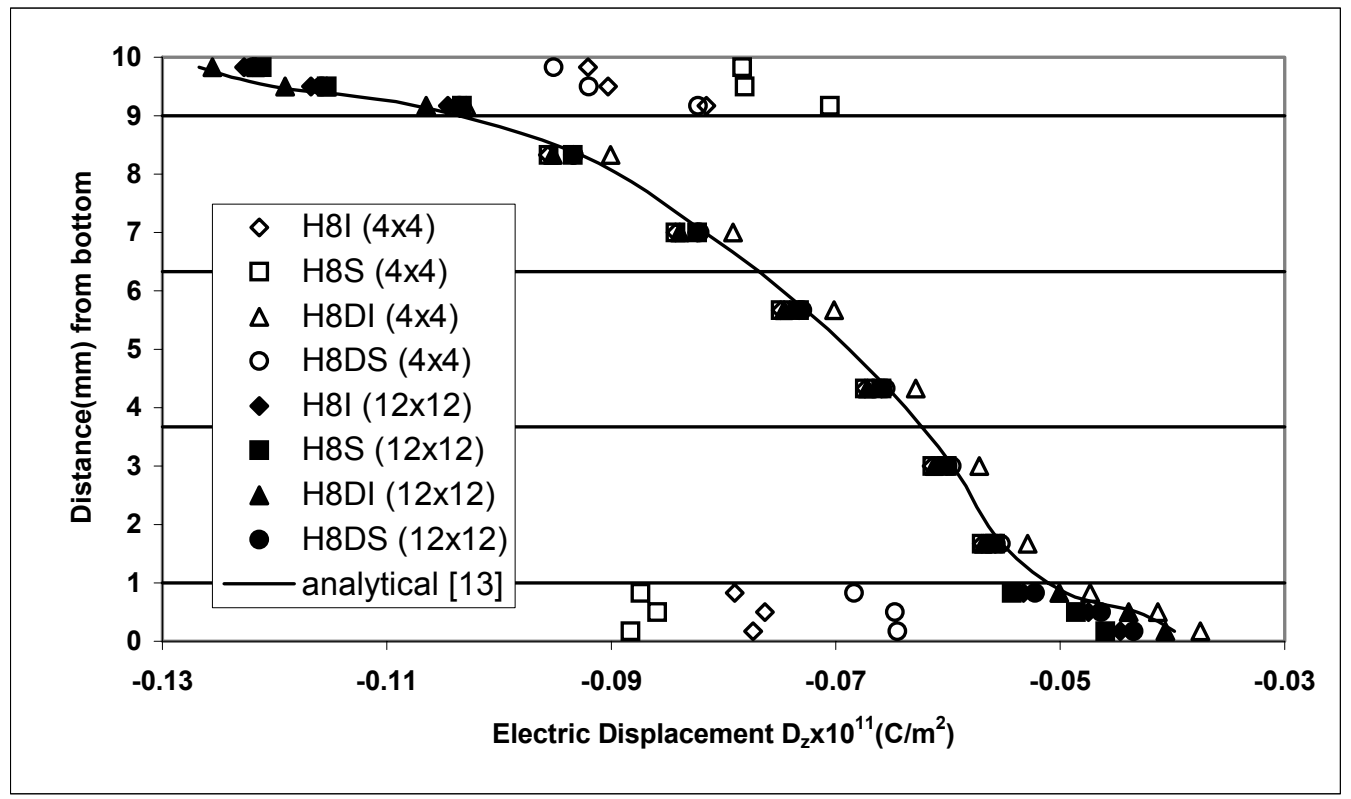

Fig.16. Variation of $D_{z}$ along AA' of the simply supported laminated plate under an applied double-sinusoidal mechanical load, see Fig.8

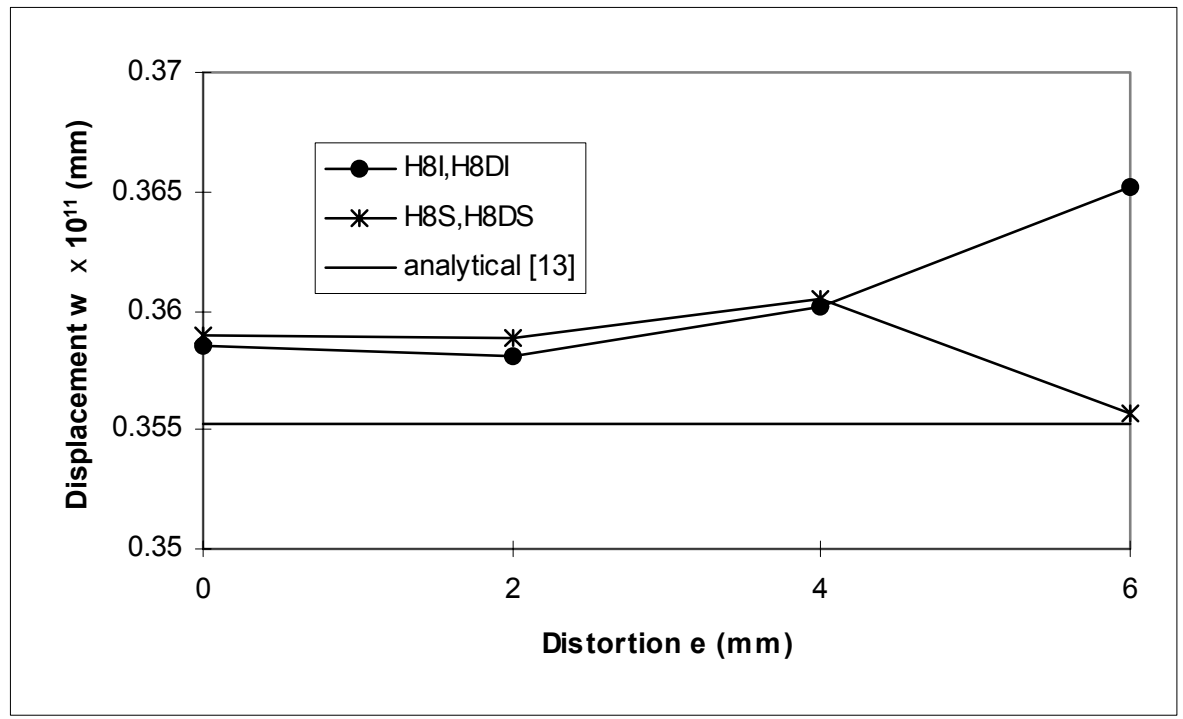

Fig.17. Effect of mesh distortion on the central vertical deflection of the simply supported laminated plate under an applied double-sinusoidal mechanical load, see Fig. 11 